\title{
Fluctuation-Dissipation Relations in the absence of Detailed Balance: formalism and applications to Active Matter
}

\author{
Sara Dal Cengio ${ }^{1}$, Demian Levis ${ }^{1,2}$, Ignacio Pagonabarraga ${ }^{1,2,3}$ \\ ${ }^{1}$ Departament de Física de la Matèria Condensada, Universitat de Barcelona, Martí i \\ Franquès 1, E08028 Barcelona, Spain \\ ${ }^{2}$ UBICS University of Barcelona Institute of Complex Systems, Martí i Franquès 1, \\ E08028 Barcelona, Spain \\ ${ }^{3}$ Centre Européen de Calcul Atomique et Moléculaire (CECAM), École \\ Polytechnique Fédérale de Lasuanne (EPFL), Batochime, Avenue Forel 2, Lausanne, \\ Switzerland \\ E-mail: sdalcengio@ub.edu
}

February 2020

\begin{abstract}
We present a comprehensive study about the relationship between the way Detailed Balance is broken in non-equilibrium systems and the resulting violations of the Fluctuation-Dissipation Theorem. Starting from stochastic dynamics with both odd and even variables under Time-Reversal, we exploit the relation between entropy production and the breakdown of Detailed Balance to establish general constraints on the non-equilibrium steady-states (NESS), which relate the non-equilibrium character of the dynamics with symmetry properties of the NESS distribution. This provides a direct route to derive extended Fluctuation-Dissipation Relations, expressing the linear response function in terms of NESS correlations. Such framework provides a unified way to understand the departure from equilibrium of active systems and its linear response. We then consider two paradigmatic models of interacting selfpropelled particles, namely Active Brownian Particles (ABP) and Active OrnsteinUhlenbeck Particles (AOUP). We analyze the non-equilibrium character of these systems (also within a Markov and a Chapman-Enskog approximation) and derive extended Fluctuation-Dissipation Relations for them, clarifying which features of these active model systems are genuinely non-equilibrium.
\end{abstract}

Submitted to: J. Stat. Mech. 


\section{Contents}

1 Introduction 3

2 Stochastic dynamics: general aspects and definitions 5

2.1 Fokker-Planck equation . . . . . . . . . . . . . . . 5

2.2 Symmetry aspects under Time-Reversal . . . . . . . . . . . . . . . . 6

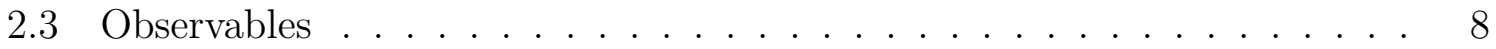

3 A preamble: Equilibrium dynamics 9

3.1 Detailed Balance . . . . . . . . . . . . . . . . . . . 9

3.2 The Fluctuation-Dissipation Theorem . . . . . . . . . . . . . . 11

4 Non-equilibrium dynamics $\mathbf{1 2}$

4.1 Quantifying the violations of Detailed Balance . . . . . . . . . . . . . 12

4.2 General constraints on Non-Equilibrium Steady-States . . . . . . . . . . 14

4.3 Extended Fluctuation-Dissipation Relations . . . . . . . . . . . . 16

5 Application to Active Particles 17

5.1 Active Brownian Particles . . . . . . . . . . . . . . 17

5.1.1 The model . . . . . . . . . . . . . . . . 17

5.1.2 Non-equilibrium character and non-interacting regime . . . . . . 17

5.1.3 Interacting regime: an effective Markovian description . . . . . . . 18

5.2 Active Ornstein-Uhlenbeck Particles . . . . . . . . . . . . . . . . . . . 20

5.2 .1 The model . . . . . . . . . . . . . . . 20

5.2 .2 Effective equilibrium regime . . . . . . . . . . . . 20

5.2.3 Non-equilibrium regime: Chapman-Enskog expansion . . . . . . . 21

6 Conclusions $\quad[23$ 


\section{Introduction}

The Fluctuation-Dissipation Theorem (FDT) relates the correlations of spontaneous fluctuations, to the fluctuations induced by external stimuli [1]. In practice, it allows to probe the response to external fields by analyzing the corresponding time-dependent equilibrium fluctuations, either in experiments or in simulations. For instance, it allows to infer transport or mechanical properties of soft materials from light scattering without ever perturbing them [2, 3]. The FDT plays a particular important role in statistical mechanics as it is among the very rare general results in non-equilibrium, although near to, conditions. It is valid for any equilibrium system (both in the classical and quantum realm) gently driven out-of-equilibrium by a small perturbation. Accordingly to the FDT, the response of an observable $A$ at time $t$ to a perturbation $h$, applied at time $s$, and causing the change in the energy of the system $E \rightarrow E-h(s) B$, is determined by an equilibrium correlation function as

$$
\left.\frac{\delta\langle A(t)\rangle}{\delta h(s)}\right|_{h \rightarrow 0}=R_{A}(t, s)=\beta \frac{\partial}{\partial s}\langle A(t) B(s)\rangle_{\mathrm{eq}}, \quad t>s
$$

where $R_{A}$ is the response function of the observable $A$ reacting to a perturbation conjugated to $B$ and $\langle A(t) B(s)\rangle_{\text {eq }}$ is the equilibrium correlation function between these two latter observables at temperature $\beta^{-1}=k_{B} T$ 国

In its general formulation above, the FDT was first derived in the context of Hamiltonian mechanics [4, 5], where the dynamics is specified via a Liouville operator and the equations of motion are invariant under Time-Reversal. It has later been extended to stochastic descriptions [6] which rely on the hypothesis of scale separation between the system of interest and the bath, the latter being a collection of (many) degrees of freedom with fast relaxation to equilibrium. Once such distinction is settled, dissipative and noisy terms enter into the equations of motion. As a result, the latter are no longer invariant under Time-Reversal. Nevertheless a footprint of reversibility holds at the stochastic description level under the name of Detailed Balance (DB) [7, 8]. As long as DB is guaranteed, the FDT holds, both in thermal and athermal states [9, 10].

For systems breaking DB, relentlessly evolving far-from-equilibrium, the FDT is no longer justified. The question of whether a similar relation as eq. (1) can be derived in this case, has been the focus of a great deal of research efforts over the last decades. In particular, several extended Fluctuation-Dissipation Relations (FDR) have been derived, using different approaches, for systems in non-equilibrium steadystates (NESS). However, contrary to equilibrium states, no universal relation such as eq. (1) exists for NESS. The establishment of a general extended FDR with the features of the equilibrium FDT, remains a central challenge towards the construction of a general framework to deal with non-equilibrium systems. In the context of stochastic dynamics, extended FDR for NESS have mostly focused on overdamped descriptions [11, 12, 13, 14, 15, 16, 17]. We refer to [18, 19, 20] for recent reviews on the topic.

$\ddagger$ We consider, without loss of generality, observables with zero mean. 
Among the variety of non-equilibrium systems, living matter constitutes a particularly interesting class. From a physics viewpoint, it can be considered as active matter: systems composed of interacting units - be it a cell, a molecular motor, a autocatalytic colloid - capable of extracting energy from their environment to perform some task and, typically (as in the cases we consider here), self-propel. In contrast with passive systems relaxing towards NESS, which are driven out-of-equilibrium by external global means (usually through their boundaries), active systems break DB at the level of each of its constituents, defining a fundamentally different class of non-equilibrium systems [21, 22].

A renewed interest in the characterization of NESS comes indeed from active matter physics. The possibility to extend equilibrium-like concepts to characterize active matter, in particular their NESS, has been the focus of intense efforts over the past decade. Most of our general understanding of such fundamental aspects of active matter has been gained through the detailed investigation of simple models of self-propelled particles, such as the Active Brownian Particles (ABP) [23, 24, 25, 26] and Active Ornstein-Uhlenbeck Particles (AOUP) [27, 28, 29, 30, 31, 32] models that we consider here. Quantities such as the pressure or chemical potential have been defined for model active systems and exploited to characterize their phase behavior and the nature of the (non-equilibrium) phase transitions they exhibit [33, 34, 35, 36, 37, 38, 39, 40, 41, 42, 43, 26, 44].

Attempts to extend the FDT to characterize the linear response of active systems has been limited to specific cases or regimes, mostly considering activity as a small parameter. In [45, 46], activity is treated as the perturbation on an otherwise equilibrium state, while in [30] a FDR is obtained in a small activity regime for which the dynamics of the system fulfills DB. In both cases, the reference state that is perturbed is not a genuine NESS: in the first case, it is an equilibrium state with Boltzmann statistics, while in the latter an effective equilibrium state with a generalized potential. The fundamental difficulties arising from the violation of DB are therefore bypassed. The linear response beyond such small activity limit has been analyzed for a single active particle in [47]. In [48], response functions were obtained beyond such limit regimes, although they are not written in terms of NESS time-correlation functions, as one wills for establishing FDR, but as weighted averages (in the spirit of Malliavin weight sampling [49]). Another strategy consists in systematically quantifying the violations of the FDT through an effective temperature [50, 51, 27, 52, 53, 54, 55, 56, 57, 58,. While this approach provides useful insights into the dynamics of NESS, it does not carry the same piece of information as a FDR, i.e. a generic way to asses the response function of an active system in terms of the steady-state fluctuations of measurable observables. Activity results on transport phenomena which are impossible in equilibrium passive systems, as recently observed in experiments involving biological microorganisms [59, 60, 61, 62] as well as artificial phoretic motors [63, 64, 65, 66. A key step towards the fundamental understanding of active materials is to characterize transport coefficients and establish extended Green-Kubo expressions resulting from the FDR. 
Here we address the question of how systems interacting active particles respond to an external small perturbation. Although the non-equilibrium nature of active systems is intrinsically different from the one of passive driven systems, as for the construction of a linear response theory, the fundamental difficulty to be tackled in both cases is the breakdown of DB. We first establish a general constraint on the NESS to be fulfilled by any Markovian dynamics, fulfilling or not DB. The framework and results obtained apply to both systems with only even variables and even and odd variables under Time-Reversal, such as overdamped and underdamped Langevin processes. Such constraint on the NESS stands for a relation between the nature of the non-equilibrium fluxes and the symmetry (under Time-Reversal) of the NESS distribution. We then derive an extended FDR for stochastic dynamics breaking DB. We finally turn to the application of these general results to archetypical models of active particles: Active Brownian Particles (ABP) and Active Ornstein-Uhlenbeck particles (AOUP). For ABP we consider the non-interacting limit and an effective equilibrium regime resulting from a Markovian approximation as discussed in [67]. For AOUP we derive a genuine, although approximated, non-equilibrium FDR unveiling the interplay between activity and interactions. We discuss in detail the specificities of AOUP as compared with $\mathrm{ABP}$ as well as the different approximation schemes used in the literature to deal with many-body effects.

The paper is organized as follows. In section 2 we establish the general framework and notation used throughout the paper. Section 3 recalls some general aspects of equilibrium dynamics that are important to clarify before moving to non-equilibrium dynamics. A reader familiar with the formalism of stochastic processes may directly move to section 4, where general aspects of non-equilibrium dynamics are discussed: We derive a general expression for the generator of the time-reversed dynamics and connect it to the concept of entropy production, allowing the derivation of general constraints a non-equilibrium stationary measure must fulfill. An extended FDR valid for systems breaking DB is then derived and discussed. Section 5 is dedicated to the application of these results to simple models of self-propelled particles (ABP and AOUP). Section 6 contains our conclusions and final remarks.

\section{Stochastic dynamics: general aspects and definitions}

\subsection{Fokker-Planck equation}

Our starting point is a generic system with $N$ dynamic variables $\boldsymbol{\Gamma} \equiv\left\{\Gamma_{i}\right\}_{i=1}^{N}$ defined on a manifold $\mathcal{M} \subset \mathbb{R}^{N}$. We introduce a probability distribution $\Psi$ which assigns $\Psi(\boldsymbol{\Gamma}, t)$ to any point $\Gamma \in \mathcal{M}$ at a time $t$. The implicit assumption is the requirement for $\Psi$ to be smooth enough for its partial derivatives to exist.

Generically the time evolution of $\Psi(\boldsymbol{\Gamma}, t)$ is described by a generator $\Omega_{0}(\boldsymbol{\Gamma})$ :

$$
\partial_{t} \Psi(\boldsymbol{\Gamma}, t)=\Omega_{0}(\boldsymbol{\Gamma}) \Psi(\boldsymbol{\Gamma}, t)
$$

together with an appropriate initial condition $\Psi(\boldsymbol{\Gamma}, 0)$. Formal integration of eq. (2) 
leads to $\Psi(\boldsymbol{\Gamma}, t)=e^{\Omega_{0} t} \Psi(\boldsymbol{\Gamma}, 0)$. We denote $\Psi_{0}$ the steady-state solution of the dynamics above, meaning

$$
\Omega_{0} \Psi_{0}=0 \text {. }
$$

Up to here, we did not need to specify the nature of the dynamics. We shall now focus on stochastic dynamics (although the following formalism could be extended to, say, Hamiltonian dynamics). In that case, an extra assumption is needed to ensure that eq. (2) is fully determined by the initial condition $\Psi(\boldsymbol{\Gamma}, 0)$ i.e. the requirement of markovianity for $\left\{\Gamma_{i}\right\}[68$. Whenever this assumption is met, the generator in eq. (2) has the so-called Fokker-Planck form:

$$
\Omega_{0}(\boldsymbol{\Gamma})=\sum_{i}\left(-\partial_{i} \mathcal{A}_{i}(\boldsymbol{\Gamma})+\sum_{j} \partial_{i} \partial_{j} \mathcal{B}_{i j}(\boldsymbol{\Gamma})\right)
$$

where $\partial_{i} \equiv \partial / \partial \Gamma_{i}, \mathcal{A} \equiv\left\{\mathcal{A}_{i}\right\}_{i=1}^{N}$ is the drift vector and $\mathcal{B} \equiv\left\{\mathcal{B}_{i j}\right\}_{i, j=1}^{N}$ is the $N \times N$ diffusion matrix. In the following, unless explicitly stated otherwise, we will take $\mathcal{B}$ to be invertible and diagonal with constant entries, such that $\mathcal{B}_{i j} \equiv D_{i} \delta_{i j}$.

The dynamics is fully specified by the knowledge of $\Psi(\boldsymbol{\Gamma}, t)$ or, equivalently, by the knowledge of the conditional probability density $P\left(\boldsymbol{\Gamma}, t \mid \boldsymbol{\Gamma}_{0}, t_{0}\right)$ defined as the probability to be in $\boldsymbol{\Gamma}$ at time $t$ given the configuration $\boldsymbol{\Gamma}_{0}$ at time $t_{0}$. Eq. (2) can be recast in terms of $P\left(\boldsymbol{\Gamma}, t \mid \boldsymbol{\Gamma}_{0}, t_{0}\right)$ as

$$
\partial_{t} P\left(\boldsymbol{\Gamma}, t \mid \boldsymbol{\Gamma}_{0}, t_{0}\right)=\Omega_{0}(\boldsymbol{\Gamma}) P\left(\boldsymbol{\Gamma}, t \mid \boldsymbol{\Gamma}_{0}, t_{0}\right)
$$

which is often called forward equation to distinguish it from the backward equation:

$$
\partial_{t_{0}} P\left(\boldsymbol{\Gamma}, t \mid \boldsymbol{\Gamma}_{0}, t_{0}\right)=-\Omega_{0}^{\dagger}\left(\boldsymbol{\Gamma}_{0}\right) P\left(\boldsymbol{\Gamma}, t \mid \boldsymbol{\Gamma}_{0}, t_{0}\right)
$$

where $\Omega_{0}^{\dagger}(\boldsymbol{\Gamma})=\sum_{i} \mathcal{A}_{i}(\boldsymbol{\Gamma}) \partial_{i}+D_{i} \partial_{i}^{2}$ is the adjoint operator of $\Omega_{0}$. The main difference between the two equations is the set of variables that we hold fix. In eq. (2) we fix the initial condition at time $t_{0}$ and we look at the evolution for $t>t_{0}$. In eq. (6), instead, we fix the final condition at time $t$ and we look at the evolution for $t_{0}<t$. This remark will show its relevance when characterizing the departure from equilibrium in systems breaking DB.

\subsection{Symmetry aspects under Time-Reversal}

We shall distinguish the dynamic variables $\Gamma_{i}$ according to their parity under TimeReversal

$$
\mathcal{T}: \Gamma \in \mathcal{M} \mapsto \varepsilon \Gamma \equiv\left\{\varepsilon_{i} \Gamma_{i}\right\} \in \mathcal{M}, \quad \quad \varepsilon_{i}= \pm 1 .
$$

Variables $\Gamma_{i}$ for which $\varepsilon_{i}=1$ are said even under Time-Reversal and variables for which $\varepsilon_{i}=-1$ are said to be odd. For instance, if one has in mind the dynamics of a particle in phase space, $\boldsymbol{\Gamma}=(x, p)$ and

$$
\mathcal{T}:(r, p) \mapsto(r,-p)
$$

The position variable $r$ is even while momentum $p$ is odd. 
The Fokker-Planck equation stands for the conservation of the probability density and can thus be written in terms of a probability flux $\boldsymbol{J} \equiv\left\{J_{i}\right\}_{i=1}^{N}$ as

$$
\begin{aligned}
& \Omega_{0} \Psi(\boldsymbol{\Gamma}, t)=-\boldsymbol{\nabla} \cdot \boldsymbol{J}(\boldsymbol{\Gamma}, t)=\partial_{t} \Psi(\boldsymbol{\Gamma}, t) \\
& J_{i}(\boldsymbol{\Gamma}, t)=\mathcal{A}_{i}(\boldsymbol{\Gamma}) \Psi(\boldsymbol{\Gamma}, t)-D_{i} \partial_{i} \Psi(\boldsymbol{\Gamma}, t)
\end{aligned}
$$

where $\boldsymbol{\nabla} \equiv\left\{\partial_{i}\right\}$. Since we allow $\left\{\Gamma_{i}\right\}$ to be either even or odd under Time-Reversal, we can decompose the drift vector in a reversible and an irreversible part, $\mathcal{A}=\mathcal{A}^{\text {rev }}+\mathcal{A}^{\mathrm{irr}}$, defined as

$$
\begin{aligned}
\mathcal{A}_{i}^{\mathrm{rev}}(\boldsymbol{\Gamma}) & \equiv \frac{1}{2}\left[\mathcal{A}_{i}(\boldsymbol{\Gamma})-\varepsilon_{i} \mathcal{A}_{i}(\varepsilon \boldsymbol{\Gamma})\right] \\
\mathcal{A}_{i}^{\mathrm{irr}}(\boldsymbol{\Gamma}) & \equiv \frac{1}{2}\left[\mathcal{A}_{i}(\boldsymbol{\Gamma})+\varepsilon_{i} \mathcal{A}_{i}(\varepsilon \boldsymbol{\Gamma})\right]
\end{aligned}
$$

which, under Time-Reversal transform as

$$
\mathcal{A}_{i}^{\mathrm{rev}}(\varepsilon \Gamma)=-\varepsilon_{i} \mathcal{A}_{i}^{\mathrm{rev}}(\Gamma), \quad \mathcal{A}_{i}^{\mathrm{irr}}(\varepsilon \Gamma)=\varepsilon_{i} \mathcal{A}_{i}^{\mathrm{irr}}(\boldsymbol{\Gamma}) .
$$

We thus identify two distinct contributions to the total probability flux $J_{i}(\boldsymbol{\Gamma}, t)=$ $J_{i}^{\mathrm{rev}}(\boldsymbol{\Gamma}, t)+J_{i}^{\mathrm{irr}}(\boldsymbol{\Gamma}, t)$, where

$$
\begin{aligned}
& J_{i}^{\mathrm{rev}}(\boldsymbol{\Gamma}, t)=\mathcal{A}_{i}^{\mathrm{rev}}(\boldsymbol{\Gamma}) \Psi(\boldsymbol{\Gamma}, t) \\
& J_{i}^{\mathrm{irr}}(\boldsymbol{\Gamma}, t)=\mathcal{A}_{i}^{\operatorname{irr}}(\boldsymbol{\Gamma}) \Psi(\boldsymbol{\Gamma}, t)-D_{i} \partial_{i} \Psi(\boldsymbol{\Gamma}, t) .
\end{aligned}
$$

We denote the steady-state flux $\boldsymbol{J}_{0}$ and define the phase-space velocity as

$$
\mathcal{V}(\boldsymbol{\Gamma}) \equiv \boldsymbol{J}_{0}(\boldsymbol{\Gamma}) / \Psi_{0}(\boldsymbol{\Gamma})=\left\{\mathcal{V}_{i}^{\mathrm{rev}}+\mathcal{V}_{i}^{\text {irr }}\right\}_{i=1}^{N}
$$

[In the following, we also report its time-dependence $\mathcal{V}(\boldsymbol{\Gamma}, t) \equiv \boldsymbol{J}(\boldsymbol{\Gamma}, t) / \Psi(\boldsymbol{\Gamma}, t)]$. The decomposition of the probability flux into two contributions with different symmetry under Time-Reversal will play a central role in the following treatment [69, 70, 71]. All the dissipative terms are embedded in eq. (15).

To illustrate the definitions above, let us consider a simple example: a Brownian particle, moving in one dimension, at position $x(t)$ and momentum $p(t)$ at time $t$, i.e. $\boldsymbol{\Gamma}=(x, p)$, described by the following Langevin equation:

$$
\dot{x}(t)=p(t) \quad \dot{p}(t)=-U^{\prime}(x)-\gamma p(t)+\sqrt{2 \gamma k_{B} T} \xi(t)
$$

where $-U^{\prime}(x)$ is the total force exerted on the particle, which can either come from inter-particle interactions or an external field, $\gamma$ is the drag coefficient and $\xi(t)$ is a Gaussian white noise of zero mean and unit variance. Here and in the rest of the paper we set the mass $m \equiv 1$. We consider here the one dimensional case for simplicity, though the extension to higher dimension is straightforward. The last two terms of the right hand side account for the coupling of the particle with a thermal bath at temperature $T$, source of noise and dissipation. In equilibrium, the FDT constraints the amplitude of the noise and dissipation to be related, as made apparent in the equation above. The latter Langevin equation, can be equivalently written as the Fokker-Planck equation, with drift vector

$$
\mathcal{A}=\left[\begin{array}{c}
\mathcal{A}_{x}=p(t) \\
\mathcal{A}_{p}=-U^{\prime}(x)-\gamma p(t)
\end{array}\right]
$$


and diffusion matrix

$$
\mathcal{B}=\left[\begin{array}{cc}
0 & 0 \\
0 & \gamma k_{B} T
\end{array}\right]
$$

Under Time-Reversal, the dynamic variables transform as

$$
\varepsilon \boldsymbol{\Gamma}=\varepsilon\left[\begin{array}{l}
x \\
p
\end{array}\right]=\left[\begin{array}{c}
x \\
-p
\end{array}\right]
$$

We now apply the definition of the reversible and irreversible drift vectors Eqs. 11, 12 and find

$$
\mathcal{A}^{\mathrm{rev}}=\left[\begin{array}{c}
p(t) \\
-U^{\prime}(x)
\end{array}\right], \mathcal{A}^{\mathrm{irr}}=\left[\begin{array}{c}
0 \\
-\gamma p(t)
\end{array}\right]
$$

In the overdamped limit, the Brownian particle can be described by

$$
\dot{x}(t)=-\mu U^{\prime}(x)+\sqrt{2 \mu k_{B} T} \xi(t)
$$

where $\mu=1 / \gamma$. The drift and diffusion vector thus read

$$
\mathcal{A}=-\mu U^{\prime}(x)=\mathcal{A}^{\mathrm{irr}}, \quad \mathcal{B}=\mu k_{B} T=D_{x} .
$$

In this case, the only dynamic variable is $x$, which is even. The absence of odd variables implies the absence of reversible fluxes, and thus $\mathcal{A}^{\text {rev }}$ is identically zero.

It is worth at this stage to make a few remarks. For the underdamped system, $\mathcal{B}$ is non-invertible. This will have a consequence on the determination of the steady-state distribution fulfilling DB as shown in the next sub-section; see therein for more details. Finally, in the absence of dissipation (and diffusion) the generator $\Omega_{0}$ would be identified with the Liouville operator. In that case, the irreversible part of the flux would vanish

and the motion would be purely reversible, as expected from Time-Reversal symmetry of the 'microscopic' Hamilton equations of motion.

\subsection{Observables}

In this section we fix some notations and definitions that will be used in the following.

Physical observables are represented by real functions acting on $\mathcal{M}$, such that $A: \Gamma \in \mathcal{M} \mapsto A(\Gamma) \in \mathbb{R}$. Their steady-state average is defined as

$$
\langle A\rangle_{0}=\int_{\mathcal{M}} d \boldsymbol{\Gamma} A(\boldsymbol{\Gamma}) \Psi_{0}(\boldsymbol{\Gamma})
$$

and the ensemble average at time $t$ is defined as

$$
\langle A\rangle_{t}=\int_{\mathcal{M}} d \boldsymbol{\Gamma} A(\boldsymbol{\Gamma}) \Psi(\boldsymbol{\Gamma}, t)=\int_{\mathcal{M}} d \boldsymbol{\Gamma} A(\boldsymbol{\Gamma}) e^{\Omega_{0} t} \Psi(\boldsymbol{\Gamma}, 0) .
$$

The time evolution may be given to the observables (instead of the probability distribution) using the adjoint of the Fokker-Planck generator

$$
\langle A\rangle_{t}=\int_{\mathcal{M}} d \boldsymbol{\Gamma} e^{\Omega_{0}^{\dagger} t} A(\boldsymbol{\Gamma}) \Psi(\boldsymbol{\Gamma}, 0) \equiv \int_{\mathcal{M}} d \boldsymbol{\Gamma} A(t) \Psi(\boldsymbol{\Gamma}, 0) .
$$




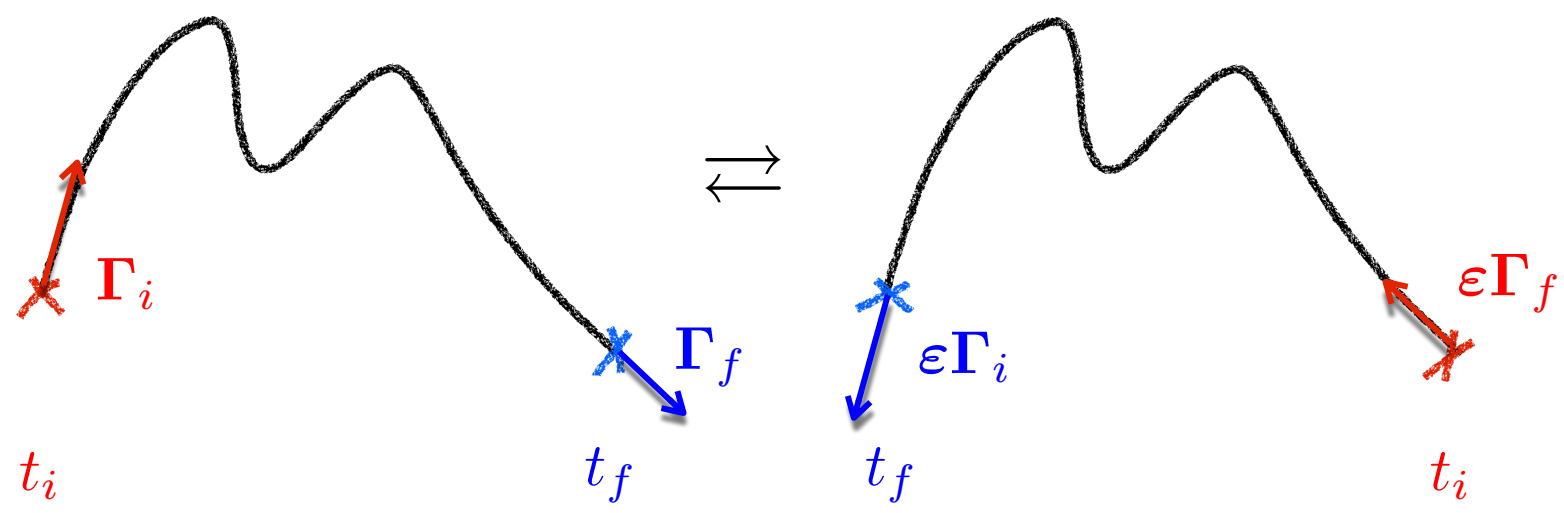

Figure 1. Illustration of a dynamics verifying Detailed Balance: the probability to transit from an initial steady-state $\boldsymbol{\Gamma}_{i}$, to a final steady-state $\boldsymbol{\Gamma}_{f}$, must be equal to the probability of the reverse transition, meaning, the probability of the transition from an initial steady-state $\boldsymbol{\epsilon} \boldsymbol{\Gamma}_{f}$ (the time-reversed version of $\boldsymbol{\Gamma}_{f}$ ), to $\boldsymbol{\epsilon} \boldsymbol{\Gamma}_{i}$.

Both expressions of $\langle A\rangle_{t}$ are fully equivalent and are respectively referred to as the Schrödinger and Heisenberg representation, by analogy with quantum mechanics [72]. In the Schrödinger the time dependence is encoded in the probability density (analogous to the wave-function), while in the Heisenberg representation, the time dependence is encoded in the observables, which are now explicitly time-dependent and are evolved by the adjoint of the evolution operator (analogous to Hermitian operators acting on a Hilbert space).

The function $R_{A}(t, s)$ encodes the linear response of an observable $A$, due to a perturbation $h$, applied at $t=0$, conjugated to an observable $B$ which results in a change of generator $\Omega_{0} \rightarrow \Omega=\Omega_{0}+\Omega_{e x t}$. It is defined as

$$
\langle A\rangle_{t}-\langle A\rangle_{0}=\int_{0}^{t} d s R_{A}(t, s) h(s)+O\left(h^{2}\right), \quad t>s .
$$

Then, writing the response function $R_{A}$ as given by the equilibrium FDT eq. 1, considering a constant perturbation $h$ and integrating by parts, we obtain

$$
\langle A\rangle_{t}-\langle A\rangle_{0}=h \beta[\langle A(t) B(t)\rangle-\langle A(t) B(0)\rangle]
$$

In its integrated version, the equilibrium FDT reduces to a simple linear relation between the integrated response and its conjugated correlation function.

\section{A preamble: Equilibrium dynamics}

\subsection{Detailed Balance}

Whether we interpret a stochastic dynamics as deriving from an underlying microscopic description fulfilling the laws of classical or quantum mechanics or not, DB constitutes the key symmetry of equilibrium. A system is said to satisfy DB if, at stationarity, any microscopic process is balanced by the reversed one. It can thus be formally written as

$$
P\left(\boldsymbol{\Gamma}_{f}, t_{f} \mid \boldsymbol{\Gamma}_{i}, t_{i}\right) \Psi_{0}\left(\boldsymbol{\Gamma}_{i}\right)=P\left(\varepsilon \boldsymbol{\Gamma}_{i}, t_{f} \mid \varepsilon \boldsymbol{\Gamma}_{f}, t_{i}\right) \Psi_{0}\left(\varepsilon \boldsymbol{\Gamma}_{f}\right)
$$


for any pair of states $\left(\boldsymbol{\Gamma}_{i}, \boldsymbol{\Gamma}_{f}\right)$ and at any times $\left(t_{i}, t_{f}\right)$ (see cartoon Fig. 1). By setting $t_{i}=t_{f}$ in eq. 30 we get:

$$
\Psi_{0}(\Gamma)=\Psi_{0}(\varepsilon \Gamma), \quad \forall \boldsymbol{\Gamma} \in \mathcal{M} .
$$

It follows that the mean value of any current-like observable, i.e. $A(\varepsilon \Gamma)=-A(\boldsymbol{\Gamma})$ must be zero if DB is fulfilled \$. Rather than being a symmetry at the level of single trajectories (as for the microscopic description), DB is formulated in eq. (31) as a symmetry property of the steady-state distribution $\Psi_{0}(\boldsymbol{\Gamma})$. Actually, a necessary and sufficient condition for DB to hold is the absence of irreversible fluxes in steady conditions [73]:

$$
\text { Detailed Balance } \Leftrightarrow \boldsymbol{J}_{0}^{\mathrm{irr}}=0
$$

where $\boldsymbol{J}_{0}^{\text {irr }}=\left\{\mathcal{A}_{i}^{\text {irr }}(\boldsymbol{\Gamma}) \Psi_{0}(\boldsymbol{\Gamma})-D_{i} \partial_{i} \Psi_{0}(\boldsymbol{\Gamma})\right\}_{i=1}^{N}$. This means that, reversible steady-state fluxes are not constrained by DB. To illustrate this aspect let us go back to the example of the Brownian particle. In the absence of even variables, i.e. in the overdamped regime, DB corresponds precisely to the absence of steady-state fluxes ( in the presence of odd (momentum-like) variables as in the underdamped description, reversible steady-state fluxes can be present in a system fulfilling DB (although physical currents must have all zero ensemble averages, see eq. (31) ) [74, 75].

The absence of irreversible fluxes can be rewritten as

$$
\partial_{i} \log \Psi_{0}(\boldsymbol{\Gamma})=D_{i}^{-1} \mathcal{A}_{i}^{\mathrm{irr}}(\boldsymbol{\Gamma}),
$$

which in turn imposes the so-called 'thermodynamic curvature' (the curl in $N$ dimensions) [76] of the irreversible drift to vanish:

$$
D_{i}^{-1} \partial_{j} \mathcal{A}_{i}^{\mathrm{irr}}(\boldsymbol{\Gamma})-D_{j}^{-1} \partial_{i} \mathcal{A}_{j}^{\mathrm{irr}}(\boldsymbol{\Gamma})=0 .
$$

The latter expression provides an alternative definition of DB in terms of geometrical properties of the drift and diffusion terms. The advantage of eq. (34) over the definition in Eqs. 32,33 is that it allows to verify if a dynamics fulfills or not DB without the need of finding $\Psi_{0}(\boldsymbol{\Gamma})$. The constraint imposed by DB on the steady-state currents provides a natural route to explicitly derive a steady solution. Whenever eq. (34) is satisfied, one can derive a steady solution by direct integration. No such a procedure exists if DB is broken, and no prescribed functional form of the multivariate Fokker-Planck equation can be derived in general. This is precisely the great advantage of equilibrium dynamics: the steady-state can be solved just by quadrature, giving the equilibrium distribution

$$
\Psi_{0}(\boldsymbol{\Gamma})=\Psi_{\text {eq }}(\boldsymbol{\Gamma})=\mathcal{N} \exp \left[\sum_{i} \int D_{i}^{-1} \mathcal{A}_{i}^{\mathrm{irr}} d \Gamma_{i}\right]
$$

with $\mathcal{N}$ the normalization constant such that $\int d \boldsymbol{\Gamma} \Psi_{0}(\boldsymbol{\Gamma})=1$.

It is straightforward to apply eq. (35) to the case of an equilibrium Brownian particle in the overdamped limit, eq. (23). In this case the integral in eq. (35) gives the $\S$ Note that here we are referring to physical currents and not to the probability current $J_{i}$ in eq. (9). Indeed, eq. (31) implies $\langle A\rangle_{0}=\int d \boldsymbol{\Gamma} A(\varepsilon \boldsymbol{\Gamma}) \Psi_{0}(\varepsilon \boldsymbol{\Gamma})=-\int d \boldsymbol{\Gamma} A(\boldsymbol{\Gamma}) \Psi_{0}(\boldsymbol{\Gamma})=-\langle A\rangle_{0}$ if $A(\varepsilon \boldsymbol{\Gamma})=-A(\boldsymbol{\Gamma})$. 
Boltzmann distribution $\Psi_{\text {eq }}(\boldsymbol{\Gamma})=\mathcal{N} \exp [-\beta U(x)]$. As we mentioned previously, a little care must be taken when applying eq. (35) to the underdamped particle of eq. (17) since in this case, the diffusive matrix is non invertible, see eq. 19. The integral over the phase space in eq. (35) has to be carried on momenta only, to find

$$
\Psi_{\text {eq }}(\boldsymbol{\Gamma}) \sim \exp \left[-\beta p^{2} / 2+\Lambda(x)\right]
$$

with $\Lambda(x)$ a function of spatial coordinates to be determined by imposing stationarity (i.e. $\Omega_{0} \Psi_{0}=0$ ). In the presence of odd variables (underdamped case), the steady-state distribution is not fully determined by $\mathrm{DB}$, which only constraints irreversible fluxes, but one has also to explicitly apply the evolution equation to specify the dependence on positions, which, as expected, results in $\Lambda=U$.

\subsection{The Fluctuation-Dissipation Theorem}

We focus now on the linear response of a system initially prepared in a steady-state. At $t=0$ we apply an infinitesimal perturbation to the drift vector $\mathcal{A} \rightarrow \mathcal{A}+\delta \mathcal{A}$. The evolution equation (2) now reads:

$$
\partial_{t} \Psi(\boldsymbol{\Gamma}, t)=\Omega \Psi(\boldsymbol{\Gamma}, t)=\left[\Omega_{0}(\boldsymbol{\Gamma})+\Omega_{\mathrm{ext}}(\boldsymbol{\Gamma})\right] \Psi(\boldsymbol{\Gamma}, t)
$$

where

$$
\Omega_{\mathrm{ext}}(\boldsymbol{\Gamma}) \Psi(\boldsymbol{\Gamma}, t)=-\boldsymbol{\nabla} \cdot[\delta \mathcal{A}(\boldsymbol{\Gamma}) \Psi(\boldsymbol{\Gamma}, t)] .
$$

accounts for the perturbation. Since the generator $\Omega$ does not explicitly depend on time, we can write [77:

$$
\int_{0}^{t} \frac{d}{d t^{\prime}} e^{\Omega t^{\prime}} d t^{\prime}=\int_{0}^{t} \Omega e^{\Omega t^{\prime}} d t^{\prime}=e^{\Omega t}-1 .
$$

We now use the latter expression into $\Psi(\boldsymbol{\Gamma}, t)=e^{\Omega t} \Psi(\boldsymbol{\Gamma}, 0)$ to obtain, to first order in $\delta \mathcal{A}$,

$$
\Psi(\boldsymbol{\Gamma}, t)=\Psi_{0}(\boldsymbol{\Gamma})+\int_{0}^{t} d t^{\prime} e^{\Omega_{0}(\boldsymbol{\Gamma}) t^{\prime}} \Omega_{\mathrm{ext}}(\boldsymbol{\Gamma}) \Psi_{0}(\boldsymbol{\Gamma}) .
$$

For an observable $A$, we then find the so-called Agarwal FDR [9]:

$$
\langle A\rangle_{t}-\langle A\rangle_{0}=\int_{0}^{t} d s\langle A(s) B(0)\rangle_{0}, \quad B(0) \equiv \frac{\Omega_{\mathrm{ext}}(\boldsymbol{\Gamma}) \Psi_{0}(\boldsymbol{\Gamma})}{\Psi_{0}(\boldsymbol{\Gamma})}
$$

where $B$ is the observable conjugated to the perturbation $\delta \mathcal{A}$ and $\langle\cdot\rangle_{0}$ is the ensemble average weighted with the initial stationary distribution $\Psi_{0}$. Alternatively, using eq. (38) into (41) the integrated response can be expressed as:

$$
\langle A\rangle_{t}-\langle A\rangle_{0}=-\int_{0}^{t} d s\left\langle A(s)\left[\nabla \cdot \delta \mathcal{A}+\delta \mathcal{A} \cdot \boldsymbol{\nabla} \log \Psi_{0}\right](0)\right\rangle_{0}
$$

which resembles the FDR as originally appeared in [78 in the context of dynamical systems. At this level, no equilibrium hypothesis has been made and, as such, Eqs. 414-42 remain valid also for NESS. For an equilibrium system fulfilling DB, i.e. $\Psi_{0}(\boldsymbol{\Gamma})=\Psi_{\text {eq }}(\boldsymbol{\Gamma})$, the conjugated observable $B$ can be computed explicitly. 


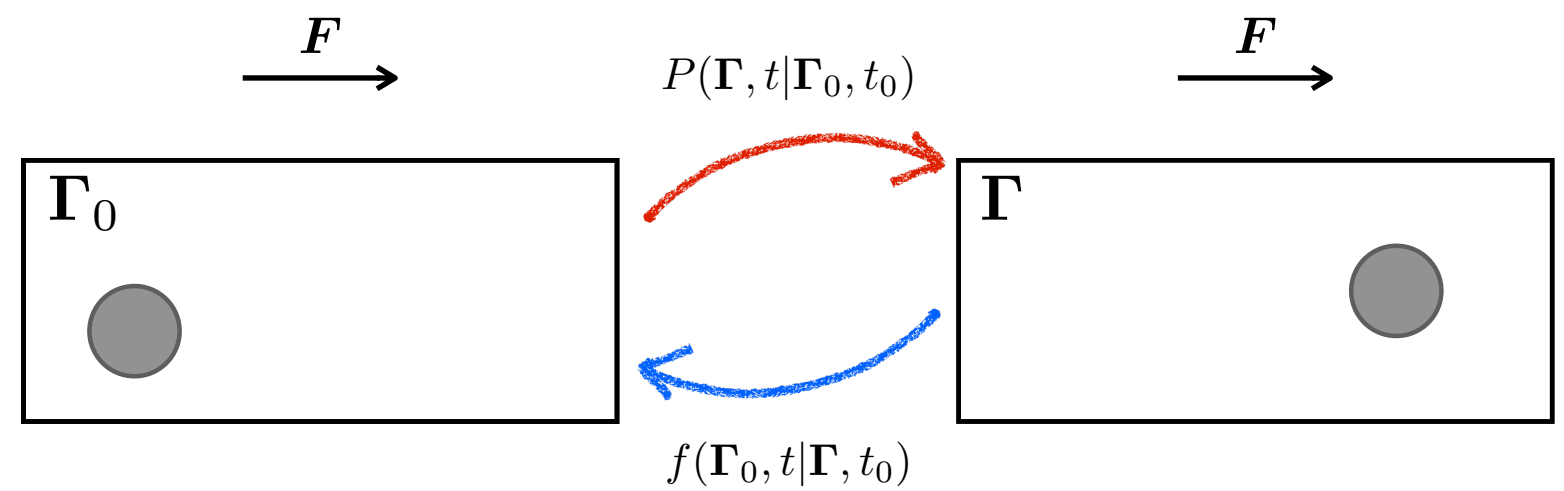

Figure 2. Illustration of the conditional probability $f$ introduced to quantify violations of DB. A force $\boldsymbol{F}$ is applied to a colloidal particle, which favors motion towards the right, and thus $f\left(\boldsymbol{\Gamma}_{0}, t \mid \boldsymbol{\Gamma}, t_{0}\right)<P\left(\boldsymbol{\Gamma}, t \mid \boldsymbol{\Gamma}_{0} t_{0}\right)$. In the absence of external drive, DB is satisfied and there is no preference to move right or left, thus $f\left(\boldsymbol{\Gamma}_{0}, t \mid \boldsymbol{\Gamma}, t_{0}\right)=P\left(\boldsymbol{\Gamma}, t \mid \boldsymbol{\Gamma}_{0} t_{0}\right)$.

As an illustration, we consider again a one-dimensional overdamped Brownian particle in contact with a thermal bath at temperature $T$ that we perturb by applying, at $t=0$, a constant external force $h$. In this case $\Omega_{0}=\mu \partial_{x}\left[k_{B} T \partial_{x}-F\right]$ with $F \equiv-U^{\prime}(x)$ the conservative force acting on the particle and $\Omega_{\text {ext }}=-\mu h \partial_{x}$. From eq. (35) we immediately obtain the Boltzmann distribution, and from eq. (41)

$$
\langle A\rangle_{t}-\langle A\rangle_{0}=-\mu \beta h \int_{0}^{t} d s\langle A(s) F(0)\rangle_{0} .
$$

If we now replace $\mu F=\dot{x}-\sqrt{2 \mu k_{B} T} \xi$ and use the fact that $R_{A}(t)=\sqrt{\beta \mu / 2}\langle A(t) \xi(0)\rangle$ [79] we find the following familiar form of the FDT for Brownian suspensions

$$
\langle A\rangle_{t}-\langle A\rangle_{0}=\beta h \int_{0}^{t} d s\langle A(s) \dot{x}(0)\rangle_{0} .
$$

From this relation, it is straightforward to derive Green-Kubo expressions for transport coefficients, such as the mobility and diffusivity. For instance, by choosing $A \equiv \dot{x}$ we find the well-known expressions

$$
\mu=\lim _{t \rightarrow \infty} \frac{\langle\dot{x}\rangle_{t}}{h}=\beta \int_{0}^{\infty} d s\langle\dot{x}(s) \dot{x}(0)\rangle_{0}, \quad D=\int_{0}^{\infty} d s\langle\dot{x}(s) \dot{x}(0)\rangle_{0} .
$$

\section{Non-equilibrium dynamics}

\subsection{Quantifying the violations of Detailed Balance}

In order to quantify the breakdown of $\mathrm{DB}$ we introduce the conditional probability to go from $\boldsymbol{\Gamma}$ to $\boldsymbol{\Gamma}_{0}$ forward in time (see Fig. 2):

$$
f\left(\boldsymbol{\Gamma}_{0}, t \mid \boldsymbol{\Gamma}, t_{0}\right) \equiv \frac{\Psi_{0}(\varepsilon \boldsymbol{\Gamma})}{\Psi_{0}\left(\boldsymbol{\Gamma}_{0}\right)} P\left(\varepsilon \boldsymbol{\Gamma}_{0}, t \mid \varepsilon \boldsymbol{\Gamma}, t_{0}\right) .
$$

Whenever DB holds $f\left(\boldsymbol{\Gamma}_{0}, t \mid \boldsymbol{\Gamma}, t_{0}\right)=P\left(\boldsymbol{\Gamma}, t \mid \boldsymbol{\Gamma}_{0} t_{0}\right)$. When DB is violated, such identification is no longer valid. Nevertheless, it is still interesting to look at the 
evolution equation for $f\left(\boldsymbol{\Gamma}_{0}, t \mid \boldsymbol{\Gamma}, t_{0}\right)$ as it allows us to quantify the breakdown of DB. Following [80] we write the backward evolution equation for $P\left(\varepsilon \boldsymbol{\Gamma}_{0}, t \mid \varepsilon \Gamma, t_{0}\right)$ :

$\partial_{t} P\left(\varepsilon \boldsymbol{\Gamma}_{0}, t \mid \varepsilon \boldsymbol{\Gamma}, t_{0}\right)=\sum_{i} \varepsilon_{i} \mathcal{A}_{i}(\varepsilon \boldsymbol{\Gamma}) \partial_{i} P\left(\varepsilon \boldsymbol{\Gamma}_{0}, t \mid \varepsilon \boldsymbol{\Gamma}, t_{0}\right)+D_{i} \partial_{i}^{2} P\left(\varepsilon \boldsymbol{\Gamma}_{0}, t \mid \varepsilon \boldsymbol{\Gamma}, t_{0}\right)$.

If we multiply both sides of the latter equation by $\Psi_{0}(\varepsilon \boldsymbol{\Gamma}) / \Psi_{0}\left(\boldsymbol{\Gamma}_{0}\right)$, and use the definition of $f\left(\boldsymbol{\Gamma}_{0}, t \mid \boldsymbol{\Gamma}, t_{0}\right)$, we obtain

$$
\partial_{t} f=\Omega_{0}(\boldsymbol{\Gamma}) f+\sum_{i} \partial_{i}\left[f\left(2 \mathcal{A}_{i}^{\mathrm{irr}}(\boldsymbol{\Gamma})-2 D_{i} \partial_{i} \log \Psi_{0}(\varepsilon \boldsymbol{\Gamma})\right]\right)
$$

where, for simplicity, we write $f\left(\boldsymbol{\Gamma}_{0}, t \mid \boldsymbol{\Gamma}, t_{0}\right) \equiv f$. When DB holds, we recover the Fokker-Planck equation: since the initial condition for $f\left(\boldsymbol{\Gamma}_{0}, t \mid \boldsymbol{\Gamma}, t_{0}\right)$ and $P\left(\boldsymbol{\Gamma}, t \mid \boldsymbol{\Gamma}_{0}, t_{0}\right)$ is the same by definition, it follows that $f=P\left(\boldsymbol{\Gamma}, t \mid \boldsymbol{\Gamma}_{0}, t_{0}\right)$, as expected.

The evolution of $f$ is generated by the time-reversal operator, $\bar{\Omega}_{0}$, defined as

$\partial_{t} f\left(\boldsymbol{\Gamma}_{0}, t \mid \boldsymbol{\Gamma}, t_{0}\right) \equiv \bar{\Omega}_{0}(\boldsymbol{\Gamma}) f\left(\boldsymbol{\Gamma}_{0}, t \mid \boldsymbol{\Gamma}, t_{0}\right)=\Psi_{0}(\varepsilon \boldsymbol{\Gamma}) \Omega_{0}^{\dagger}(\varepsilon \boldsymbol{\Gamma})\left[f\left(\boldsymbol{\Gamma}_{0}, t \mid \boldsymbol{\Gamma}, t_{0}\right) \Psi_{0}(\varepsilon \boldsymbol{\Gamma})^{-1}\right]$.

Its adjoint

$$
\bar{\Omega}_{0}^{\dagger}(\boldsymbol{\Gamma})=\Psi_{0}(\varepsilon \Gamma)^{-1} \Omega_{0}(\varepsilon \Gamma) \Psi_{0}(\varepsilon \Gamma),
$$

has a precise dynamical meaning: it evolves (forward in time) the observable $A$ along time-reversal paths, i.e.

$$
\langle A\rangle_{(-t)} \equiv \int d \boldsymbol{\Gamma} \Psi_{0}(\boldsymbol{\Gamma}) e^{\bar{\Omega}^{\dagger} t} A(\boldsymbol{\Gamma}), \quad t>0
$$

generalizing the formulation of Baiesi and Maes [19] to systems with odd variables. The above definition of the time-reversal operator and the expression of its adjoint in terms of the original generator, do not rely on any assumption concerning the steady-state distribution. From eq. (50), one easily finds the following expression quantifying the breakdown of DB in terms of irreversible probability fluxes:

$$
\frac{\Omega_{0}^{\dagger}(\boldsymbol{\Gamma})-\bar{\Omega}_{0}^{\dagger}(\boldsymbol{\Gamma})}{2}=\frac{\varepsilon \boldsymbol{J}_{0}^{\mathrm{irr}}(\varepsilon \boldsymbol{\Gamma})}{\Psi_{0}(\varepsilon \boldsymbol{\Gamma})} \cdot \nabla \equiv \varepsilon \mathcal{V}^{\mathrm{irr}}(\varepsilon \boldsymbol{\Gamma}) \cdot \nabla .
$$

It immediately follows that DB holds if and only if the time-reversal operator equals the original generator of the dynamics

$$
\text { Detailed Balance } \Leftrightarrow \quad \Omega_{0}(\boldsymbol{\Gamma})=\bar{\Omega}_{0}(\boldsymbol{\Gamma}) \text {. }
$$

Since DB only constraints irreversible fluxes to vanish, it is thus expected that its breakdown only concerns their presence. The status of DB as a symmetry of the dynamics is now transparent. These expressions are general and can be exploited to quantify the breakdown of DB in systems with both odd and even variables, such as collections of interacting particles with inertia [81] or noisy RLC circuits [82]. 


\subsection{General constraints on Non-Equilibrium Steady-States}

It is useful to notice that the irreversible phase-space velocity quantifying the nonequilibrium character of the dynamics, or breakdown of $\mathrm{DB}$, is directly related to the housekeeping entropy production, i.e. the entropy being produced in a NESS. For a stochastic dynamics described by the Fokker-Planck equation (9 10), the housekeeping entropy production rate is expressed as [88]:

$$
\left\langle\dot{S}_{\mathrm{hk}}\right\rangle_{t}=k_{B} \sum_{i} \int d \boldsymbol{\Gamma} D_{i}^{-1} \Psi(\boldsymbol{\Gamma}, t)\left(\varepsilon \mathcal{V}_{i}^{\mathrm{irr}}(\varepsilon \boldsymbol{\Gamma})\right)^{2} \geq 0
$$

where we recognize inside the integral the phase space velocity quantifying the violations of DB. The house-keeping term constitutes the entropy production necessary to sustain a NESS and it is positive as soon as DB is broken. It may be compared with the standard formula for the total entropy production [83, 84, 88]:

$$
\left\langle\dot{S}_{\text {tot }}\right\rangle_{t}=k_{B} \sum_{i} \int d \boldsymbol{\Gamma} D_{i}^{-1} \Psi(\boldsymbol{\Gamma}, t)\left(\mathcal{V}_{i}^{\text {irr }}(\boldsymbol{\Gamma}, t)\right)^{2} \geq 0 .
$$

embedding contributions coming from the breakdown of DB as well as from any time-dependent processes (the so-called excess entropy production). By definition, in

stationary conditions, $\dot{S}_{\mathrm{hk}}$ must reduce to the total entropy change $\dot{S}_{\text {tot }}$, since no timedependent contribution is present. While this is obvious for even variables only, in presence of odd variables this observation provides a general constraint on the phasespace velocity such that:

$$
\left[\mathcal{V}_{i}^{\text {irr }}(\varepsilon \Gamma)\right]^{2}=\left[\mathcal{V}_{i}^{\text {irr }}(\boldsymbol{\Gamma})\right]^{2}
$$

This constitutes one of our main results: as we will show below, this equality constraints any NESS and allows to relate the nature of the irreversible fluxes, responsible for the breakdown of DB, with the symmetry of the NESS distribution $\Psi_{0}$.

In order to illustrate this general result and its consequences, we now consider a paradigmatic non-equilibrium model: a one-dimensional particle in a periodic potential $U$, coupled to a thermal bath and driven out-of-equilibrium by a non-conservative force $F \|$. The Langevin equation governing the dynamics of such system is (see Fig. (2))

$$
\dot{x}(t)=p(t) \quad \dot{p}(t)=-U^{\prime}(x)+F-\gamma p(t)+\sqrt{2 \gamma k_{B} T} \xi(t) .
$$

In this simple case, the two components of irreversible part of the phase-space velocity which enter in eq. 56 read

$$
\begin{aligned}
& \mathcal{V}_{x}^{\operatorname{irr}}(x, p)=0 \\
& \mathcal{V}_{p}^{\operatorname{irr}}(x, p)=-\gamma p-\gamma k_{B} T \frac{\partial}{\partial p} \log \Psi_{0}(x, p) .
\end{aligned}
$$

As we already mentioned, $\Psi_{0}$ does not necessary have a given parity under TimeReversal. Nevertheless (without lack of generality) we may rewrite it as:

$$
\Psi_{0}(x, p)=\mathcal{N} \exp (-\Phi(x, p))=\mathcal{N} \exp \left[-\left(\Phi_{+}+\Phi_{-}\right)\right]
$$

|| Periodicity is needed in order to guarantee the existence of a steady-state. 
where we have decomposed the generalized potential $\Phi$ into an even and odd contribution: $\Phi(x,-p)_{ \pm}= \pm \Phi(x, p)$ | Then, the general constraint eq. (56) yields

$$
\begin{aligned}
& \Phi_{+}(x, p)=\beta \frac{p^{2}}{2}+\Lambda(x) \\
& \mathcal{V}_{p}^{\operatorname{irr}}(x, p)=\gamma k_{B} T \frac{\partial \Phi_{-}}{\partial p}
\end{aligned}
$$

where $\Lambda(x)$ is any derivable function of $x$. Thus, the even part of the generalized potential must be of the latter form, meaning that even terms $p^{2 n}$ with $n>1$ are strictly forbidden in the NESS distribution. As expected, the dependence of $\Psi(x, p)$ on the positions, which are associated to reversible fluxes, is not constrained at all. Instead, irreversible fluxes are given by (gradients of) the odd part of the generalized potential. Thus, eq. (56) establishes a generic, and explicit, relationship between irreversible fluxes and the symmetry of the NESS distribution.

In the limit $U \rightarrow 0$, the NESS distribution of this simple model can be computed analytically and consists in a 'tilted' Gaussian distribution in $p$ and a uniform distribution in $x$, such as :

$$
\Psi_{0}(x, p)=\mathcal{N} \exp \left[-\frac{(p-\langle p\rangle)^{2}}{2 k_{B} T}\right]
$$

where $\langle p\rangle=F / \gamma$ is the mean velocity of the particle. Combining eq. 63) with eq. 62 we find $\Phi_{-}=-p F / \gamma k_{B} T$ and consequently:

$$
\begin{aligned}
\mathcal{V}_{p}^{\text {irr }} & =-F \\
\left\langle\dot{S}_{\mathrm{hk}}\right\rangle_{0} & =\frac{F^{2}}{\gamma T}>0
\end{aligned}
$$

which reveals the non-equilibrium character of this system and the nature of its phase space velocity: it is proportional to the particle net current, resulting in a positive house-keeping entropy production.

Now turning back into a more general discussion, eq. (56) can be rewritten in terms of the generalized potential as

$$
\left[\mathcal{A}_{i}^{\mathrm{irr}}+D_{i} \partial_{i} \Phi_{+}\right] D_{i} \partial_{i} \Phi_{-}=0 \quad \forall i
$$

which, in order to be verified, requires one of the two following set of conditions:

$$
\partial_{i} \Phi_{+}(\boldsymbol{\Gamma})=-D_{i}^{-1} \mathcal{A}_{i}^{\mathrm{irr}}(\boldsymbol{\Gamma}) \quad \text { or } \quad \partial_{i} \Phi_{-}=0 .
$$

Note that if both constraints are fulfilled simultaneously then the system obeys DB [see eq. (33)]. Eq. (56) 'relaxes' one of the two constraints, allowing to distinguish two different ways of breaking DB, namely

$$
\begin{aligned}
& \quad \Phi_{-}(\boldsymbol{\Gamma})=0 \text { but } \partial_{i} \Phi_{+}(\boldsymbol{\Gamma}) \neq-D_{i}^{-1} \mathcal{A}_{i}^{\mathrm{irr}}(\boldsymbol{\Gamma}) \\
& \text { or } \\
& \qquad \partial_{i} \Phi_{+}(\boldsymbol{\Gamma})=-D_{i}^{-1} \mathcal{A}_{i}^{\mathrm{irr}}(\boldsymbol{\Gamma}) \text { but } \Phi_{-}(\boldsymbol{\Gamma}) \neq 0 .
\end{aligned}
$$

I The generalized potential is assumed to be a differential function of the dynamic variables. 
Any non-equilibrium system with only even variables falls into the first category. On the contrary, systems with odd variables, such as the driven underdamped Brownian particle considered above or the Active Ornstein-Ulhenbeck Particles that we treat in detail in section 5.2, fall into the second category. For systems with odd variables, it follows that the irreversible flux is directly related to the odd part of the NESS distribution, such that:

$$
\mathcal{V}_{i}^{\mathrm{irr}}(\boldsymbol{\Gamma})=D_{i} \partial_{i} \Phi_{-}(\boldsymbol{\Gamma})
$$

\subsection{Extended Fluctuation-Dissipation Relations}

We now consider a system initially prepared in a NESS with probability distribution $\Psi_{0}$. At time $t=0$, we apply a perturbation such that $\mathcal{A} \rightarrow \mathcal{A}+\delta \mathcal{A}$. Although the Agarwal FDR eq. (41) remains valid, the lack of knowledge on $\Psi_{0}$ does not allow us to derive an explicit expression of the response function in terms of NESS correlations. To move further, an option would be to provide a reliable scheme to approximate $\Psi_{0}$. We follow this strategy in the next section when dealing with active particles. However, some general expressions can be establish through the NESS properties derived above. For instance, alternative FDRs can be written in a way that explicitly relate the nonequilibrium response of the system to the symmetry properties of $\Psi_{0}$.

The Agarwal FDR can be rewritten in terms of the even and odd part of the NESS distribution as

$$
\begin{aligned}
\langle A\rangle_{t}-\langle A\rangle_{0}= & \sum_{i}\left\{-\int_{0}^{t} d s\left\langle A(s)\left(\partial_{i} \delta \mathcal{A}_{i}\right)(0)\right\rangle_{0}+\int_{0}^{t} d s\left\langle A(s)\left(\delta \mathcal{A}_{i} \partial_{i} \Phi_{+}\right)(0)\right\rangle_{0}\right. \\
& \left.+\int_{0}^{t} d s\left\langle A(s)\left(\delta \mathcal{A}_{i} \partial_{i} \Phi_{-}\right)(0)\right\rangle_{0}\right\}
\end{aligned}
$$

We then make use of the relations, derived in the previous section, between the generalized potential and the phase space velocity to establish the following extended FDR

$$
\begin{aligned}
\langle A\rangle_{t}-\langle A\rangle_{0}= & \sum_{i}\left\{-\int_{0}^{t} d s\left\langle A(s)\left(\partial_{i} \delta \mathcal{A}_{i}\right)(0)\right\rangle_{0}-D_{i}^{-1} \int_{0}^{t} d s\left\langle A(s)\left(\delta \mathcal{A}_{i} \mathcal{A}_{i}^{\mathrm{irr}}\right)(0)\right\rangle_{0}\right. \\
& \left.+D_{i}^{-1} \int_{0}^{t} d s\left\langle A(s)\left(\delta \mathcal{A}_{i} \mathcal{V}_{i}^{\mathrm{irr}}\right)(0)\right\rangle_{0}\right\}
\end{aligned}
$$

which is valid both for overdamped and underdamped systems in the presence any type of external perturbation $\delta \mathcal{A}$. As such, it generalizes the FDR appearing in $[12,19,89$, derived for an overdamped particle subjected to a conservative perturbation $\delta \mathcal{A} \equiv-\mu \partial_{x} U$, for which it reduces to:

$$
\langle A\rangle_{t}-\langle A\rangle_{0}=D_{x}^{-1} \mu \int_{0}^{t} d s\left(\langle A(s) \dot{U}(0)\rangle_{0}-\left\langle A(s) \partial_{x} U(0) \mathcal{V}_{x}^{\text {irr }}(0)\right\rangle_{0}\right)
$$

and if we consider the perturbation to be a constant force of amplitude $h, U \equiv h x$, we end up with the non-equilibrium extension of the FDT eq. (44) as derived by Speck and 
Seifert [12]:

$$
\langle A\rangle_{t}-\langle A\rangle_{0}=D_{x}^{-1} \mu h \int_{0}^{t} d s\left(\langle A(s) \dot{x}(0)\rangle_{0}-\left\langle A(s) \mathcal{V}_{x}^{\mathrm{irr}}(0)\right\rangle_{0}\right) .
$$

The term comprising the irreversible phase space velocity is responsible for the nonequilibrium character of the dynamics as it encodes the breakdown of DB. We notice by comparing (71) and (72) that in the presence of odd variables, full knowledge on $\Psi_{0}$ is not required to determine the response, but only on its odd part under Time-Reversal.

\section{Application to Active Particles}

\subsection{Active Brownian Particles}

5.1.1. The model We consider now $N$ two-dimensional overdamped Active Brownian Particles (ABP) moving in two-dimensional space. They self-propelled with a constant velocity $v_{0}$, along their orientation $\boldsymbol{n}_{i}=\left(\cos \left(\theta_{i}\right), \sin \left(\theta_{i}\right)\right)$ and obey the following set of coupled Langevin equations

$$
\dot{\boldsymbol{r}}_{i}(t)=\mu_{0} \boldsymbol{F}_{i}+v_{0} \boldsymbol{n}_{i}(t)+\boldsymbol{\xi}_{i}(t), \dot{\theta}_{i}(t)=\nu_{i}(t)
$$

where $\boldsymbol{F}_{i}=-\partial U / \partial \boldsymbol{r}_{i}, \mu_{0}$ is the single particle mobility and $\boldsymbol{\xi}$ and $\nu$ are zeromean Gaussian noises verifying $\left\langle\boldsymbol{\xi}_{i}(t) \boldsymbol{\xi}_{j}\left(t^{\prime}\right)\right\rangle=2 \mu_{0} k_{B} T \delta_{i j} \delta\left(t-t^{\prime}\right) \mathbf{1}$ and $\left\langle\nu_{i}(t) \nu_{j}\left(t^{\prime}\right)\right\rangle=$ $2 D_{\theta} \delta_{i j} \delta\left(t-t^{\prime}\right)$. It follows that

$$
\left\langle\boldsymbol{n}_{i}(t) \cdot \boldsymbol{n}_{i}(0)\right\rangle=e^{-D_{\theta} t},
$$

defining a persistence time $\tau=1 / D_{\theta}$. We define the Péclet number $\mathrm{Pe}=v_{0} / \sigma D_{\theta}$ where $\sigma$ is a characteristic length-scale set, for instance, by the inter-particle potential. Equilibrium is recovered both in the limit of $v_{0} \rightarrow 0$ or $\tau \rightarrow 0$. In both cases, the departure from equilibrium can be quantified by Pe. The generator, or Fokker-Planck operator, corresponding to this Langevin dynamics is

$$
\Omega_{0}(\boldsymbol{\Gamma})=\sum_{i} \frac{\partial}{\partial \boldsymbol{r}_{i}}\left(\mu_{0} k_{B} T \frac{\partial}{\partial \boldsymbol{r}_{i}}-\mu_{0} \boldsymbol{F}_{i}-v_{0} \boldsymbol{n}_{i}\right)+D_{\theta} \sum_{i} \frac{\partial^{2}}{\partial \theta_{i}^{2}}
$$

where $\boldsymbol{\Gamma} \equiv\left\{\boldsymbol{r}_{i}, \theta_{i}\right\}$ : All dynamical variables in ABP are considered even under TimeReversal.

\subsubsection{Non-equilibrium character and non-interacting regime For $\mathrm{ABP}, \mathrm{DB}$ is fullfilled} if and only if

$$
\left\{\begin{array}{l}
\left(\mu_{0} \boldsymbol{F}_{i}+v_{0} \boldsymbol{n}_{i}\right) \Psi_{0}(\boldsymbol{\Gamma})=\mu_{0} k_{B} T \frac{\partial}{\partial \boldsymbol{r}_{i}} \Psi_{0}(\boldsymbol{\Gamma}) \\
\frac{\partial}{\partial \theta_{i}} \Psi_{0}(\boldsymbol{\Gamma})=0
\end{array}\right.
$$

These two equations can not simultaneously hold due to the self-propulsion term, and therefore, ABP generically break DB. By integrating the first condition, we get $\log \Psi_{0} \sim-\beta\left[U-\sum_{i} v_{0} \boldsymbol{n}_{i} \cdot \boldsymbol{r}_{i} / \mu_{0}\right]$. However, the second condition imposes $\Psi_{0}$ to be a function of positions only, which is inconsistent with the first condition because of the 
term in $\boldsymbol{n}_{i} \cdot \boldsymbol{r}_{i}$. In the passive case, $v_{0} \rightarrow 0$, DB is recovered together with the standard Boltzmann distribution.

An illustrative example for which we can explicitly compute the phase space velocity

is a free ABP. The Fokker-Plank generator reads $\Omega_{0}=\left[\partial_{\boldsymbol{r}}\left(\mu_{0} k_{B} T \partial_{\boldsymbol{r}}-v_{0} \boldsymbol{n}\right)+D_{\theta} \frac{\partial^{2}}{\partial \theta^{2}}\right]$. A stationary solution of the Fokker-Planck equation can be derived $\Psi_{0}(\boldsymbol{r}, \theta)=\rho_{0} / 2 \pi$ [90]. The phase space velocity corresponding to this homogeneous NESS is

$$
\mathcal{V}^{\text {irr }}(t)=\left(\mathcal{V}_{\boldsymbol{r}}^{\text {irr }}, \mathcal{V}_{\theta}^{\text {irr }}\right)=\left(v_{0} \boldsymbol{n}(t), 0\right)
$$

Since the system is overdamped, there are no reversible fluxes. In order to apply the extended FDR, we consider a constant force perturbation $h$ applied along the $x$-axis. By choosing $A=\dot{x}$ in eq. (73) we obtain

$$
\frac{\langle\dot{x}\rangle_{t}}{h}=\beta \int_{0}^{t} d s\langle\dot{x}(s) \dot{x}(0)\rangle_{0}-\beta v_{0} \int_{0}^{t} d s\langle\dot{x}(s) \cos \theta(0)\rangle_{0}
$$

resulting in the following extended Stokes-Einstein relation in the long time regime

$$
D / \mu_{0}=k_{B} T+\frac{v_{0}^{2}}{2 D_{\theta} \mu_{0}} .
$$

Therefore, non-interacting ABP fulfill the Stokes-Einstein relation with an effective temperature

$$
T_{\text {eff }} / T=1+\frac{v_{0}^{2}}{2 D_{\theta} \mu_{0} k_{B} T}
$$

It is worth noting here that, although ABP generically break DB in a fundamental way (and do not allow for a zero current steady-state solution), in the non-interacting limit they admit a NESS which fulfills the Stokes-Einstein relation.

5.1.3. Interacting regime: an effective Markovian description As as evidenced by the extended FDR eq. (72), the response of a non-equilibrium system is not completely determined by NESS correlations of physical observables, but also depends on the specific form of its phase space velocity. In other to establish explicit FDR for interacting ABP one can approximate the dynamics by an effective equilibrium one that fulfills DB. Such kind of approximation has been used for ABP and also AOUP and come under different names, the most usual ones being Unified Colored Noise and Fox approximation [91, 92, 29, 93]. In both cases, the steady-state distribution does not correspond to the equilibrium Boltzmann distribution in terms of the energy function of the original dynamics, but a 'Boltzmann-like' distribution in terms of an effective energy function generating the approximated dynamics. Despite the non-Boltzmann character of the steady-state distribution resulting from these approaches, all the difficulties associated with the absence of DB are lifted and one can readily derive a FDR by direct application of the general results presented in the previous section.

To be more specific, we turn now into the analysis of interacting ABP within the Fox approximation [94, 95], as we previously presented in [67]. The starting point is to integrate out the angular variables appearing in the ABP dynamics. As usual, the 
integration of some stochastic variables introduces memory in the dynamics, here in the form of a colored noise with correlation time $\tau$. The equations of motion (75) can be approximated by

$$
\dot{\boldsymbol{r}}_{i}(t)=\mu_{0} \boldsymbol{F}_{i}+\boldsymbol{\eta}_{i}(t)
$$

where the noise $\boldsymbol{\eta}_{i}$ is approximately Gaussian with zero mean and variance $\left\langle\boldsymbol{\eta}_{i}(t) \boldsymbol{\eta}_{j}(s)\right\rangle=\left(2 \mu_{0} k_{B} T \delta(t-s)+v_{0}^{2} e^{-|t-s| / \tau} / 2\right) \delta_{i j} \mathbf{1}$. The ABP dynamics in the reduced configuration space $\tilde{\boldsymbol{\Gamma}} \equiv\left\{\boldsymbol{r}_{i}\right\}$ is approximated by an effective Fokker-Planck dynamics generated by the operator :

$$
\Omega_{0}^{M}(\tilde{\boldsymbol{\Gamma}})=\sum_{\alpha} \partial_{\alpha}\left(\sum_{\beta} \partial_{\beta} \mathcal{D}_{\beta \alpha}(\tilde{\boldsymbol{\Gamma}})-\mu_{0} F_{\alpha}(\tilde{\boldsymbol{\Gamma}})\right)
$$

where $\mathcal{D} \equiv\left\{\mathcal{D}_{\beta \alpha}\right\}$ is an effective $2 N \times 2 N$ diffusivity tensor and where greek indices run over the spatial coordinates and the particle labels. To first order in $\tau \mu_{0} \partial_{\alpha} F_{\beta}$, it reads

$$
\mathcal{D}_{\alpha \beta}(\tilde{\boldsymbol{\Gamma}})=\mu_{0} k_{B} T \delta_{\alpha \beta}+\frac{v_{0}^{2} \tau}{2}\left(\delta_{\alpha \beta}+\tau \mu_{0} \partial_{\alpha} F_{\beta}\right) .
$$

Note that the Fox approximation is meaningful only when $\left|\tau \mu_{0} \partial_{\alpha} F_{\beta}\right|<1$. As we show below, this effective dynamics fulfills DB. The first step is to write the condition of DB eq. (32) for the stationary probability density $\Psi_{0}(\tilde{\boldsymbol{\Gamma}})$ :

$$
\sum_{\beta} \mathcal{D}_{\beta \alpha}(\tilde{\boldsymbol{\Gamma}}) \partial_{\beta} \Psi_{0}(\tilde{\boldsymbol{\Gamma}})=\Psi_{0}(\tilde{\boldsymbol{\Gamma}})\left(\mu_{0} F_{\alpha}-\sum_{\beta} \partial_{\beta} \mathcal{D}_{\beta \alpha}(\tilde{\boldsymbol{\Gamma}})\right)
$$

We then multiply both sides by $\mathcal{D}_{\alpha \gamma}^{-1}$ and sum over the index $\alpha$ to get:

$$
\partial_{\gamma} \log \Psi_{0}(\tilde{\boldsymbol{\Gamma}})=\sum_{\alpha} \mathcal{D}_{\alpha \gamma}^{-1}(\tilde{\boldsymbol{\Gamma}})\left[\mu_{0} F_{\alpha}-\sum_{\beta} \partial_{\beta} \mathcal{D}_{\beta \alpha}(\tilde{\boldsymbol{\Gamma}})\right] \equiv \beta F_{\gamma}^{\mathrm{eff}}(\tilde{\boldsymbol{\Gamma}})
$$

Using eq. (85) and eq. (87) the effective force may we re-expressed as [93]:

$$
\beta F_{\gamma}^{\mathrm{eff}}(\tilde{\boldsymbol{\Gamma}})=\frac{\mu_{0}}{D_{a}} F_{\gamma}(\tilde{\boldsymbol{\Gamma}})-\left(\frac{\mu_{0} v_{0} \tau}{2 D_{a}}\right)^{2} \sum_{\alpha} \partial_{\gamma}\left(F_{\alpha}(\tilde{\boldsymbol{\Gamma}})\right)^{2}-\partial_{\gamma} \log [\operatorname{det} \mathcal{D}(\tilde{\boldsymbol{\Gamma}})]
$$

where $D_{a} \equiv \mu_{0} k_{B} T+v_{0}^{2} \tau / 2$. It is now straightforward to verify that $\partial_{\beta} F_{\gamma}^{\mathrm{eff}}-\partial_{\gamma} F_{\beta}^{\mathrm{eff}}=0$. As a result, the system fulfills DB and therefore the FDT. This in turn implies that $\boldsymbol{F}^{\mathrm{eff}}$ derives from an effective potential, such that an analytical expression for $\Psi_{0}$ in terms of an effective energy function can be derived [29].

Before leaving this section, it is worth mentioning that a diagonal-Laplacian approximation for $\mathcal{D}(\tilde{\boldsymbol{\Gamma}})$ was recently introduced and verified a posteriori [96, 97, 98, 93]. Within this approximation the effective force $\boldsymbol{F}_{i}^{\text {eff }}$ on particle $i$ reads:

$$
\boldsymbol{F}_{i}^{\mathrm{eff}}(\tilde{\boldsymbol{\Gamma}})=k_{B} T\left(\mu_{0} \boldsymbol{F}_{i}-\partial_{i} \mathcal{D}_{i}\right) / \mathcal{D}_{i}
$$

with

$$
\mathcal{D}_{i}(\tilde{\boldsymbol{\Gamma}})=D_{a}\left(\frac{1}{1-\tau \mu_{0} \partial_{i} \cdot \boldsymbol{F}_{i}}\right)
$$

where $\partial_{i} \cdot \boldsymbol{F}_{i}=\partial_{x_{i}} F_{i}^{x}+\partial_{y_{i}} F_{i}^{y}$, further simplifying the analysis of ABP within the Fox approximation. 


\subsection{Active Ornstein-Uhlenbeck Particles}

5.2.1. The model We consider in this section a similar model of self-propelled particles, now governed by the following set of two-dimensional overdamped Langevin equations

$$
\begin{aligned}
& \dot{\boldsymbol{r}}_{i}(t)=\mu_{0} \boldsymbol{F}_{i}+\boldsymbol{v}_{i} \\
& \dot{\boldsymbol{v}}_{i}(t)=-\frac{\boldsymbol{v}_{i}}{\tau}+\sqrt{\frac{2 D_{0}}{\tau^{2}}} \boldsymbol{\eta}_{i}(t)
\end{aligned}
$$

where $\boldsymbol{F}_{i} \equiv-\partial U / \partial \boldsymbol{r}_{i}$ is a conservative force acting on particle $i$ (whose origin can be interactions with other particles or an external potential) and $\boldsymbol{v}_{i}$ is the fluctuating self-propulsion velocity which is described by an Ornstein-Uhlenbeck process with characteristic persistence time $\tau$. Self-propulsion introduces persistence in the spatio-temporal dynamics of the active particles via the autocorrelation function of the self-propulsion velocity $\left\langle\boldsymbol{v}_{i}(t) \boldsymbol{v}_{j}\left(t^{\prime}\right)\right\rangle=D_{0} / \tau e^{-\left|t-t^{\prime}\right| / \tau} \delta_{i j} \mathbf{1}$ and reduces to passive (equilibrium) Brownian motion in the limit $\tau \rightarrow 0$, for which $\left\langle\boldsymbol{v}_{i}(t) \boldsymbol{v}_{j}\left(t^{\prime}\right)\right\rangle \rightarrow 2 D_{0} \delta(t-$ $\left.t^{\prime}\right) \delta_{i j} \mathbf{1}$. Although a standard thermal noise could be added into the Langevin equation of AOUP, such contribution is assumed to be small with respect to the active noise $\boldsymbol{v}$ and might be considered redundant, as it is not needed to recover equilibrium. These AOUP can be thought of as an approximate treatment of the ABP dynamics. Indeed, the reduced ABP dynamics obtained from the integration of the angular variables eq. (83) can be identified, in the absence of translational noise $(T=0)$, to the AOUP dynamics by setting $v_{0}^{2} / 2$ (in $\mathrm{ABP}$ ) to $D_{0} / \tau$ (in AOUP).

Although originally thought of as an overdamped process, eq. (91) involves velocity variables $\boldsymbol{v}$ that can be considered as being odd under Time-Reversal. Following this interpretation, eq. (91) can be rewritten as an underdamped Langevin process [30]

$$
\begin{aligned}
& \dot{\boldsymbol{r}}_{i}(t)=\boldsymbol{p}_{i} \\
& \dot{\boldsymbol{p}}_{i}(t)=\mu_{0}\left(\sum_{j} \boldsymbol{p}_{j} \cdot \partial_{j}\right) \boldsymbol{F}_{i}-\frac{\boldsymbol{p}_{i}}{\tau}+\mu_{0} \frac{\boldsymbol{F}_{i}}{\tau}+\sqrt{\frac{2 D_{0}}{\tau^{2}}} \boldsymbol{\eta}_{i}(t)
\end{aligned}
$$

where $\partial_{i} \equiv \partial / \partial \boldsymbol{r}_{i}$. The corresponding generator reads

$$
\Omega_{0}(\boldsymbol{\Gamma})=\sum_{i}\left[-\boldsymbol{p}_{i} \cdot \partial_{i}-\partial_{\boldsymbol{p}_{i}} \cdot\left(\mu_{0}\left(\sum_{j} \boldsymbol{p}_{j} \cdot \partial_{j}\right) \boldsymbol{F}_{i}-\frac{\boldsymbol{p}_{i}}{\tau}+\mu_{0} \frac{\boldsymbol{F}_{i}}{\tau}-\frac{D_{0}}{\tau^{2}} \partial_{\boldsymbol{p}_{i}}\right)\right]
$$

where $\partial_{\boldsymbol{p}_{i}} \equiv \partial / \partial \boldsymbol{p}_{i}$ and $\boldsymbol{\Gamma}=\left\{\boldsymbol{r}_{i}, \boldsymbol{p}_{i}\right\}$.

5.2.2. Effective equilibrium regime For the AOUP model, the DB condition eq. (32) reduces to:

$$
\frac{1}{\tau^{2}} \partial_{\boldsymbol{p}_{i}} \log \Psi_{0}(\boldsymbol{\Gamma})=\beta\left(\sum_{j} \boldsymbol{p}_{j} \cdot \partial_{j}\right) \boldsymbol{F}_{i}-\frac{\boldsymbol{p}_{i}}{D_{0} \tau}
$$

Its formal solution can be expressed up to a function $\Lambda\left(\left\{\boldsymbol{r}_{i}\right\}\right)$ that only depend on space variables such as

$$
\Psi_{0}=\exp \left[-\Lambda\left(\left\{\boldsymbol{r}_{i}\right\}\right)-\frac{\beta \tau^{2}}{2}\left(\sum_{i} \boldsymbol{p}_{i} \cdot \partial_{i}\right)^{2} U-\sum_{i} \frac{\tau}{D_{0}} \frac{\boldsymbol{p}_{i}^{2}}{2}\right]
$$


where $\beta \equiv \mu_{0} / D_{0}$. In order for DB to hold, the system must fulfill the following equation

$$
\sum_{i}\left[\partial_{i} \Lambda+\frac{\beta \tau^{2}}{2}\left(\sum_{j} \boldsymbol{p}_{j} \cdot \partial_{j}\right)^{2} \partial_{i} U-\frac{\beta^{2} D_{0} \tau}{2} \partial_{i} \sum_{j}\left|\partial_{j} U\right|^{2}-\beta \partial_{i} U\right] \Psi_{0}=0 .
$$

This equation does not have a solution because of the second term comprising a $p$-dependence. Interestingly, for a potential with vanishing third derivatives the latter term vanishes and an exact 'equilibrium' solution exists [30, 32]:

$$
\Psi_{\text {eq }}=\mathcal{N} \exp \left[-\beta U-\frac{\tau}{2} \sum_{i}\left(\frac{\boldsymbol{p}_{i}^{2}}{D_{0}}+\beta^{2} D_{0}\left|\partial_{i} U\right|^{2}\right)-\frac{\beta \tau^{2}}{2}\left(\sum_{i} \boldsymbol{p}_{i} \cdot \partial_{i}\right)^{2} U\right]
$$

We wrote equilibrium in quotes because, contrarily to the standard Boltzmann measure, the probability of a given configuration is not solely given by $e^{-\beta U}$, but by a more complicated function, also involving $\boldsymbol{p}$. This form is not a priori obvious from the mere inspection of the generator of the microscopic dynamics. [The same remark holds for ABP within the Fox approximation discussed earlier, as the effective potential eq. 88 can hardly be guessed from the original Fokker-Planck equation.] However, in this equilibrium-like regime, AOUP fulfill DB, there are no irreversible fluxes, and the FDT holds. An equilibrium solution also exists in the case of non-interacting particles $U=0$ for which AOUP are formally equivalent to an ideal gas of underdamped particles. In all other cases, for a generic $U$, the model breaks DB and therefore falls out-of-equilibrium.

5.2.3. Non-equilibrium regime: Chapman-Enskog expansion An approximated stationary distribution $\Psi_{0}$ for AOUP, beyond its equilibrium-like regime, has recently been derived via the Chapman-Enskog expansion by Bonilla [32. Our aim being to study the impact of activity on the response of an interacting system, we briefly present the Chapman-Enskog results as appeared in [32] and use them to establish extended FDR for AOUP.

The Chapman-Enskog expansion constitutes a standard perturbative approach to derive the Navier-Stokes equation from the Boltzmann equation [99, 100]. It is based on the notions of local equilibrium and time scale separation. The latter is accounted for by the introduction of a small parameter $\epsilon=\ell / L$ defined as the ratio between a microscopic and a macroscopic characteristic length. In kinetic theory, $\ell$ is typically the mean free path between collisional events and $L$ the size of the system. Likewise, we may associate to the AOUP two different scales: a microscopic one associated to the persistence time $\tau$ and diffusive length $\sqrt{D_{0} \tau}$ (characterizing the local persistence due to activity), and a mesoscopic one associated to the inter-particle interactions, with a 'slow' characteristic time $\tau_{0}$ and a large characteristic length $L$. We introduce the ratio parameter $\epsilon \equiv \frac{\sqrt{D_{0} \tau}}{L} \equiv \frac{\tau}{\tau_{0}}$ and rescale the AOUP equations of motion Eqs. 93 94 according to $t \equiv t / \tau_{0}, \boldsymbol{r} \equiv \boldsymbol{r} / L$ and $\boldsymbol{F} \equiv \boldsymbol{F} L / \beta^{-1}$. The Fokker-Planck equation of AOUP can thus be written in the following non-dimensional form

$$
\sum_{i} \partial_{\boldsymbol{p}_{i}} \cdot\left(\boldsymbol{p}_{i}+\partial_{\boldsymbol{p}_{i}}\right) \Psi=\epsilon \partial_{t} \Psi+\epsilon \sum_{i}\left[\boldsymbol{p}_{i} \cdot \partial_{i}+\boldsymbol{F}_{i} \cdot \partial_{\boldsymbol{p}_{i}}+\epsilon \partial_{\boldsymbol{p}_{i}} \cdot\left(\sum_{j} \boldsymbol{p}_{j} \cdot \partial_{j}\right) \boldsymbol{F}_{i}\right] \Psi(100)
$$


From here, the idea is to carry on a perturbative expansion in $\epsilon$. For $\epsilon=0$, a solution of eq. 100 is:

$$
\Psi^{(\epsilon=0)}(\boldsymbol{\Gamma})=\frac{e^{-\sum_{i} \boldsymbol{p}_{i}^{2} / 2}}{(2 \pi)^{N}} R(\boldsymbol{r}, t)
$$

where $R(\boldsymbol{r}, t)$ is the normalized marginal density such that $\int \Pi_{i} d \boldsymbol{r}_{i} R(\boldsymbol{r}, t)=1$. For a system with strong time-scale separation, $\epsilon \ll 1$, we assume that the functional dependence on $\left\{\boldsymbol{p}_{i}\right\}$ and $R$ in eq. (101) is preserved, and expand the probability distribution as a power series in $\epsilon$ :

$$
\Psi(\boldsymbol{\Gamma})=\frac{e^{-\sum_{i} \boldsymbol{p}_{i}^{2} / 2}}{(2 \pi)^{N}} R(\boldsymbol{r}, t ; \epsilon)+\sum_{j} \epsilon^{j} \phi^{(j)}(\boldsymbol{\Gamma}, R) .
$$

The crucial assumption of the Chapman-Enskog method is to still interpret $R$ in 102 as the marginal distribution embedding the spatiotemporal dependence upon integration over the velocities. This corresponds to impose for $\phi^{(j)}$ :

$$
\int \Pi_{i} d \boldsymbol{p}_{i} \phi^{(j)}(\boldsymbol{\Gamma}, R)=0 \quad \forall j .
$$

The ansatz eq. (102) is inserted into eq. (100), resulting in a hierarchy of equations for the various terms in the expansion. We solve the set of equations up to $\sim o\left(\epsilon^{3}\right)$ and obtain the following (now made dimensional) probability distribution [30, 32]:

$$
\begin{aligned}
\Psi_{0}(\boldsymbol{\Gamma}) & \simeq \mathcal{N} \exp \left[-\beta U-\sum_{i} \frac{\tau}{2}\left(\frac{\boldsymbol{p}_{i}^{2}}{D_{0}}+\beta^{2} D_{0}\left(\partial_{i} U\right)^{2}-3 \beta D_{0} \partial_{i}^{2} U\right)\right. \\
& \left.-\frac{\tau^{2}}{2}\left(\beta\left(\sum_{j} \boldsymbol{p}_{j} \cdot \partial_{j}\right)^{2} U+\beta D_{0} \sum_{i, j}\left(\boldsymbol{p}_{j} \cdot \partial_{j}\right) \partial_{i}^{2} U\right)+\frac{\tau^{3}}{6} \beta\left(\sum_{j} \boldsymbol{p}_{j} \cdot \partial_{j}\right)^{3} U\right] .
\end{aligned}
$$

Some details on the derivation of eq. (104) are given in the Appendix for the one dimensional case. The generalization to higher dimensions is straightforward but lengthy (see also [32] for further details).

We are now in the position of computing the non-equilibrium response of AOUP up to third order in $\epsilon$. First, let us decompose $\Psi_{0}$ into its symmetric and antisymmetric parts:

$$
\begin{aligned}
& \Phi_{+}=\beta U+\sum_{i} \frac{\tau}{2}\left(\frac{\boldsymbol{p}_{i}^{2}}{D_{0}}+\beta^{2} D_{0}\left(\partial_{i} U\right)^{2}-3 \mu_{0} \partial_{i}^{2} U\right)+\frac{\tau^{2}}{2} \beta\left(\sum_{j} \boldsymbol{p}_{j} \cdot \partial_{j}\right)^{2} U \\
& \Phi_{-}=\sum_{i, j} \frac{\tau^{2}}{2} \beta D_{0}\left(\boldsymbol{p}_{j} \cdot \partial_{j}\right) \partial_{i}^{2} U-\frac{\tau^{3}}{6} \beta\left(\sum_{i} \boldsymbol{p}_{i} \cdot \partial_{i}\right)^{3} U
\end{aligned}
$$

Note that, indeed $\Phi_{+}$does not contain powers of $p$ larger than 2 , as forbidden by the non-equilibrium constraint of eq. (56). The signature of the departure from equilibrium is all embedded in $\Phi_{-}$, being different from zero (see eq. (69)). Particularly, the latter 
vanishes for a quadratic potential, as expected from the discussion in the previous section.

We now apply a constant force $h$ along the $x$-axis on a tagged particle $n$. In this case, the extended FDR eqs. (71,72) reads

$$
\langle A\rangle_{t}-\langle A\rangle_{0}=\delta \mathcal{A}_{p_{n}}\left[-\left(D_{p_{n}}\right)^{-1} \int_{0}^{t} d s\left\langle A(s) \mathcal{A}_{p_{n}}^{\mathrm{ir}}(0)\right\rangle_{0}+\int_{0}^{t} d s\left\langle A(s) \frac{\partial \Phi_{-}}{\partial p_{n}^{x}}(0)\right\rangle_{0}\right]
$$

where $\delta \mathcal{A}_{p_{n}}=\mu_{0} h / \tau$ and $D_{p_{n}}=D_{0} / \tau^{2}$, leading to

$$
\langle A\rangle_{t}-\langle A\rangle_{0}=h\left[-\tau \beta \int_{0}^{t} d s\left\langle A(s) \mathcal{A}_{p_{n}}^{\mathrm{irr}}(0)\right\rangle_{0}+\frac{\mu_{0}}{\tau} \int_{0}^{t} d s\left\langle A(s) \frac{\partial \Phi_{-}}{\partial p_{n}^{x}}(0)\right\rangle_{0}\right] .
$$

The first term is directly determined upon making the identification $\mathcal{A}_{p_{n}}^{\mathrm{irr}}=\mu_{0}\left(\sum_{j} \boldsymbol{p}_{j}\right.$. $\left.\partial_{j}\right) F_{n}^{x}-p_{n}^{x} / \tau$. The second integral requires the knowledge of the odd-symmetric part of $\Psi_{0}$, which, to third order in $\epsilon$ is given by eq. (106). All in all, we derive the following FDR for AOUP

$$
\begin{aligned}
\langle A\rangle_{t}-\langle A\rangle_{0} & =\beta h\left[\int_{0}^{t} d s\left\langle A(s) p_{n}^{x}(0)\right\rangle_{0}-\tau \mu_{0} \int_{0}^{t} d s\left\langle A(s)\left(\sum_{j} \boldsymbol{p}_{j} \cdot \partial_{j}\right) F_{n}^{x}(0)\right\rangle_{0}\right. \\
& \left.-\frac{1}{2} \mu_{0} \tau D_{0}\left(\int_{0}^{t} d s\left\langle A(s) \frac{\partial}{\partial x_{n}}\left(\sum_{j} \partial_{j} \cdot \boldsymbol{F}_{j}\right)(0)\right\rangle_{0}-\frac{\tau}{D_{0}} \int_{0}^{t} d s\left\langle A(s)\left(\sum_{j} \boldsymbol{p}_{j} \cdot \partial_{j}\right)^{2} F_{n}^{x}(0)\right\rangle_{0}\right)\right]
\end{aligned}
$$

By choosing $A \equiv \boldsymbol{p}_{n}$ we eventually obtain an extended Stokes-Einstein relation:

$$
\begin{aligned}
\mu & =\beta\left[D-\tau \mu_{0} \int_{0}^{\infty} d s\left\langle p_{n}^{x}(s)\left(\sum_{j} \boldsymbol{p}_{j} \cdot \partial_{j}\right) F_{n}^{x}(0)\right\rangle_{0}\right. \\
& \left.-\frac{1}{2} \mu_{0} \tau D_{0}\left(\int_{0}^{\infty} d s\left\langle p_{n}^{x}(s) \frac{\partial}{\partial x_{n}}\left(\sum_{j} \partial_{j} \cdot \boldsymbol{F}_{j}\right)(0)\right\rangle_{0}-\frac{\tau}{D_{0}} \int_{0}^{\infty} d s\left\langle p_{n}^{x}(s)\left(\sum_{j} \boldsymbol{p}_{j} \cdot \partial_{j}\right)^{2} \boldsymbol{F}_{n}(0)\right\rangle_{0}\right)\right]
\end{aligned}
$$

where $D$ is the many-body diffusivity $D=\int_{0}^{\infty} d s\left\langle p_{n}^{x}(s) p_{n}^{x}(0)\right\rangle$. The expression above embeds the violations to the usual Stokes-Einstein relation due to the interplay between activity and inter-particle interactions, up to order $o\left(\tau^{2}\right)$. In the absence of interactions the Stokes-Einstein relation is restored. This is also true for the case of a harmonic potential $U(r)=k|\boldsymbol{r}|^{2} / 2$, for which we are left with

$$
\mu=\beta^{\mathrm{eff}} \int_{0}^{t} d s\left\langle p_{n}^{x}(0) p_{n}^{x}(s)\right\rangle_{0}
$$

being $\beta^{\text {eff }}=\beta\left(1+\mu_{0} \tau k\right)$ an effective temperature which depends on the stiffness of the external potential [27]. In contrast with ABP, the existence of a Stokes-Einstein relation for a harmonic potential in AOUP is due to the fact that the model fulfills DB.

\section{Conclusions}

In this paper we have recalled, and discussed in detail, the pivotal role played by Detailed Balance as the defining feature of equilibrium dynamics, and how its breakdown outof-equilibrium can be quantified by the presence of irreversible steady-state fluxes. 
We have analyzed the symmetry properties of such fluxes under Time-Reversal for systems with odd and even variables, taking as illustrative examples Langevin processes describing Brownian particles both in the underdamped and overdamped regimes. We have developed a general formalism based on Fokker-Planck operators that allows us to express irreversible steady-fluxes in terms of the difference between the generator of the time-reversed dynamics and the original one.

By making the connection between the breakdown of Detailed Balance and the different contributions to the entropy production, we derived a constraint of the irreversible steady-state fluxes. This general result applies to non-equilibrium systems and provides non-trivial information for systems with dynamic variables which are odd under Time-Reversal. In particular, it constraints the functional dependence of the NESS distribution on its odd variables. We then considered the linear response of a system in a NESS and derived extended Fluctuation-Dissipation Relations, allowing to express non-equilibrium response functions as NESS correlations and shedding light upon the nature of the different terms responsible for violations of the equilibrium Fluctuation-Dissipation Theorem.

We then apply these general results and formalism to Active Brownian Particles (ABP) and Active Ornstein-Uhlenbeck Particles (AOUP). While ABP generically break Detailed Balance, AOUP fulfill Detailed Balance in the dilute limit, or in the case of a harmonic potential. The non-equilibrium nature of these two model systems is therefore not equivalent. We then analyze their linear response in the presence of generic many-body interactions in an approximated fashion. In the case of ABP, we recall the Markov approximation method due to Fox and show that the effective dynamics resulting from it fulfills Detailed Balance, and therefore also the standard FluctuationDissipation Theorem. For AOUP we exploit the Chapman-Enskog expansion performed in [32] which allows to derive an extended Fluctuation-Dissipation Relation beyond its effective equilibrium regime. We discuss the violations of the Stokes-Einstein relation in these models of active particles and show the possibility of quantifying them in terms of effective temperatures.

Although some of the results presented here were known, as the existence of an effective equilibrium regime of AOUP and the NESS solution obtained from the Chapman-Enskog expansion, the discussion about the violations of DB and the FDT were scattered and scarce. The extended FDR, as well as the connection with the parity of the NESS distribution and the constraint on the phase-space velocity we derived, enrich previous discussions on non-equilibrium response, clarify the non-equilibrium nature of active model systems and provide a set of analytic results that should be of interest to study non-equilibrium systems in general, well beyond the context of active systems. 


\section{Acknowledgments}

We warmly thank Jorge Kurchan, Matteo Polettini and Patrick Pietzonka for useful discussions and suggestions. S.D.C. acknowledges funding from the European Union's Horizon 2020 Framework Programme/European Training Programme 674979 NanoTRANS. D.L. acknowledges MCIU/AEI/FEDER for financial support under grant agreement RTI2018-099032-J-I00. I.P. acknowledges support from Ministerio de Ciencia, Innovación y Universidades (Grant No. PGC2018-098373-B-100 AEI/FEDER-EU) and from Generalitat de Catalunya under project 2017SGR-884, and Swiss National Science Foundation Project No. 200021-175719.

\section{References}

[1] Ryogo Kubo, Morikazu Toda, and Natsuki Hashitsume. Statistical physics II: nonequilibrium statistical mechanics, volume 31. Springer Science \& Business Media, 1991.

[2] Wyn Brown. Dynamic light scattering: the method and some applications, volume 313. Clarendon Press Oxford, 1993.

[3] Luca Cipelletti and Laurence Ramos. Slow dynamics in glassy soft matter. Journal of Physics: Condensed Matter, 17(6):R253, 2005.

[4] Ryogo Kubo. Statistical-mechanical theory of irreversible processes. i. general theory and simple applications to magnetic and conduction problems. Journal of the Physical Society of Japan, 12(6):570-586, 1957.

[5] Ryogo Kubo. The fluctuation-dissipation theorem. Reports on progress in physics, 29(1):255, 1966.

[6] Peter Hnggi and Harry Thomas. Stochastic processes: Time evolution, symmetries and linear response. Physics Reports, 88(4):207 - 319, 1982.

[7] Hannes Risken. The fokker-planck equation. Springer, 1996.

[8] Robert Graham and Hermann Haken. Generalized thermodynamic potential for markoff systems in detailed balance and far from thermal equilibrium. Zeitschrift für Physik A Hadrons and nuclei, 243(3):289-302, 1971.

[9] Girish Saran Agarwal. Fluctuation-dissipation theorems for systems in non-thermal equilibrium and applications. Zeitschrift für Physik A Hadrons and nuclei, 252(1):25-38, 1972.

[10] Hermann Haken. Exact stationary solution of a fokker-planck equation for multimode laser action including phase locking. Zeitschrift für Physik A Hadrons and nuclei, 219(3):246-268, Jun 1969.

[11] Takahiro Harada and Shin-ichi Sasa. Equality connecting energy dissipation with a violation of the fluctuation-response relation. Physical Review Letters, 95:130602, Sep 2005.

[12] Thomas Speck and Udo Seifert. Restoring a fluctuation-dissipation theorem in a nonequilibrium steady state. EPL (Europhysics Letters), 74(3):391, 2006.

[13] Jacques Prost, Jean-Franois Joanny, and Juan M. R. Parrondo. Generalized fluctuationdissipation theorem for steady-state systems. Physical Review Letters, 103(9):090601, 2009.

[14] Marco Baiesi, Christian Maes, and Bram Wynants. Fluctuations and response of nonequilibrium states. Physical Review Letters, 103(1):010602, 2009.

[15] Raphaël Chetrite and Krzysztof Gawędzki. Eulerian and lagrangian pictures of non-equilibrium diffusions. Journal of Statistical Physics, 137(5):890, Aug 2009.

[16] Udo Seifert and Thomas Speck. Fluctuation-dissipation theorem in nonequilibrium steady states. EPL (Europhysics Letters), 89(1):10007, 2010.

[17] Jorge Kurchan. Fluctuation theorem for stochastic dynamics. Journal of Physics A: Mathematical and General, 31(16):3719-3729, apr 1998. 
[18] Umberto Marini Bettolo Marconi, Andrea Puglisi, Lamberto Rondoni, and Angelo Vulpiani. Fluctuation-dissipation: response theory in statistical physics. Physics reports, 461(4-6):111$195,2008$.

[19] Marco Baiesi and Christian Maes. An update on the nonequilibrium linear response. New Journal of Physics, 15(1):013004, 2013.

[20] Alessandro Sarracino and Angelo Vulpiani. On the fluctuation-dissipation relation in nonequilibrium and non-hamiltonian systems. Chaos: An Interdisciplinary Journal of Nonlinear Science, 29(8):083132, 2019.

[21] Michael E. Cates. Diffusive transport without detailed balance in motile bacteria: does microbiology need statistical physics? Reports on Progress in Physics, 75(4):042601, 2012.

[22] Clemens Bechinger, Roberto Di Leonardo, Hartmut Löwen, Charles Reichhardt, Giorgio Volpe, and Giovanni Volpe. Active particles in complex and crowded environments. Reviews of Modern Physics, 88:045006, Nov 2016.

[23] Pawel Romanczuk, Markus Bär, Werner Ebeling, Benjamin Lindner, and Lutz Schimansky-Geier. Active brownian particles. The European Physical Journal Special Topics, 202(1):1-162, 2012.

[24] Yaouen Fily and Maria Cristina Marchetti. Athermal phase separation of self-propelled particles with no alignment. Physical Review Letters, 108(23):235702, 2012.

[25] Gabriel S. Redner, Michael F. Hagan, and Aparna Baskaran. Structure and dynamics of a phaseseparating active colloidal fluid. Physical Review Letters, 110:055701, Jan 2013.

[26] Pasquale Digregorio, Demian Levis, Antonio Suma, Leticia F. Cugliandolo, Giuseppe Gonnella, and Ignacio Pagonabarraga. Full phase diagram of active brownian disks: From melting to motility-induced phase separation. Physical Review Letters, 121:098003, Aug 2018.

[27] Grzegorz Szamel. Self-propelled particle in an external potential: Existence of an effective temperature. Physical Review E, 90(1):012111, 2014.

[28] Claudio Maggi, Matteo Paoluzzi, Nicola Pellicciotta, Alessia Lepore, Luca Angelani, and Roberto Di Leonardo. Generalized energy equipartition in harmonic oscillators driven by active baths. Physical Review Letters, 113(23):238303, 2014.

[29] Umberto Marini Bettolo Marconi and Claudio Maggi. Towards a statistical mechanical theory of active fluids. Soft matter, 11(45):8768-8781, 2015.

[30] Étienne Fodor, Cesare Nardini, Michael E. Cates, Julien Tailleur, Paolo Visco, and Frédéric van Wijland. How far from equilibrium is active matter? Physical Review Letters, 117(3):038103, 2016.

[31] Lennart Dabelow, Stefano Bo, and Ralf Eichhorn. Irreversibility in active matter systems: Fluctuation theorem and mutual information. Physical Review X, 9(2):021009, 2019.

[32] Luis L. Bonilla. Active ornstein-uhlenbeck particles. Physical Review E, 100(2):022601, 2019.

[33] Sho C. Takatori, Wen Yan, and John F. Brady. Swim pressure: stress generation in active matter. Physical Review Letters, 113(2):028103, 2014.

[34] Stewart A. Mallory, Andela Šarić, Chantal Valeriani, and Angelo Cacciuto. Anomalous thermomechanical properties of a self-propelled colloidal fluid. Physical Review E, 89(5):052303, 2014.

[35] Félix Ginot, Isaac Theurkauff, Demian Levis, Christophe Ybert, Lydéric Bocquet, Ludovic Berthier, and Cécile Cottin-Bizonne. Nonequilibrium equation of state in suspensions of active colloids. Physical Review X, 5(1):011004, 2015.

[36] Alexandre P. Solon, Yaouen Fily, Aparna Baskaran, Michael E. Cates, Kardar Mehran Kafri, Yariv, and Julien Tailleur. Pressure is not a state function for generic activefluids. Nature Physics, 11(8):673-678, 2015.

[37] Roland G. Winkler, Adam Wysocki, and Gerhard Gompper. Virial pressure in systems of spherical active brownian particles. Soft matter, 11(33):6680-6691, 2015.

[38] Raphael Wittkowski, Adriano Tiribocchi, Joakim Stenhammar, Rosalind J. Allen, Davide Marenduzzo, and Michael E. Cates. Scalar $\varphi 4$ field theory for active-particle phase separation. Nature communications, 5(1):1-9, 2014. 
[39] Joakim Stenhammar, Davide Marenduzzo, Rosalind J. Allen, and Michael E. Cates. Phase behaviour of active brownian particles: the role of dimensionality. Soft Matter, 10:1489-1499, 2014.

[40] Demian Levis, Joan Codina, and Ignacio Pagonabarraga. Active brownian equation of state: metastability and phase coexistence. Soft Matter, 13:8113-8119, 2017.

[41] Siddharth Paliwal, Jeroen Rodenburg, René van Roij, and Marjolein Dijkstra. Chemical potential in active systems: predicting phase equilibrium from bulk equations of state? New Journal of Physics, 20(1):015003, 2018.

[42] Stefano Steffenoni, Gianmaria Falasco, and Klaus Kroy. Microscopic derivation of the hydrodynamics of active-brownian-particle suspensions. Physical Review E, 95(5):052142, 2017.

[43] Alexandre P. Solon, Joakim Stenhammar, Michael E. Cates, Yariv Kafri, and Julien Tailleur. Generalized thermodynamics of motility-induced phase separation: phase equilibria, laplace pressure, and change of ensembles. New Journal of Physics, 20(7):075001, 2018.

[44] Sophie Hermann, Daniel de las Heras, and Matthias Schmidt. Non-negative interfacial tension in phase-separated active brownian particles. Physical Review Letters, 123:268002, Dec 2019.

[45] Abhinav Sharma and Joseph M. Brader. Communication: Green-kubo approach to the average swim speed in active brownian systems. The Journal of Chemical Physics, 145(16):161101, 2016.

[46] Alexander Liluashvili, Jonathan Ónody, and Thomas Voigtmann. Mode-coupling theory for active brownian particles. Physical Review E, 96(6):062608, 2017.

[47] Lorenzo Caprini, Umberto Marini Bettolo Marconi, and Angelo Vulpiani. Linear response and correlation of a self-propelled particle in the presence of external fields. Journal of Statistical Mechanics: Theory and Experiment, 2018(3):033203, 2018.

[48] Kiryl Asheichyk, Alexandre P. Solon, Christian M. Rohwer, and Matthias Krüger. Response of active brownian particles to shear flow. The Journal of Chemical Physics, 150(14):144111, 2019.

[49] Patrick B. Warren and Rosalind J. Allen. Malliavin weight sampling for computing sensitivity coefficients in brownian dynamics simulations. Physical Review Letters, 109(25):250601, 2012.

[50] Leticia F. Cugliandolo. The effective temperature. Journal of Physics A: Mathematical and Theoretical, 44(48):483001, 2011.

[51] Davide Loi, Stefano Mossa, and Leticia F. Cugliandolo. Effective temperature of active matter. Physical Review E, 77(5):051111, 2008.

[52] Demian Levis and Ludovic Berthier. From single-particle to collective effective temperatures in an active fluid of self-propelled particles. EPL (Europhysics Letters), 111(6):60006, 2015.

[53] Eyal Ben-Isaac, YongKeun Park, Gabriel Popescu, Frank LH. Brown, Nir S. Gov, and Yair Shokef. Effective temperature of red-blood-cell membrane fluctuations. Physical Review Letters, 106(23):238103, 2011.

[54] Leticia F. Cugliandolo, Giuseppe Gonnella, and Isabella Petrelli. Effective temperature in active brownian particles. Fluctuation and Noise Letters, 18(02):1940008, 2019.

[55] Sarah Eldeen, Ryan Muoio, Paris Blaisdell-Pijuan, Ngoc La, Mauricio Gomez, Alex Vidal, and Wylie Ahmed. Quantifying the non-equilibrium activity of an active colloid. Soft Matter, 2020.

[56] Suvendu Mandal, Benno Liebchen, and Hartmut Löwen. Motility-induced temperature difference in coexisting phases. Physical Review Letters, 123(22):228001, 2019.

[57] Elijah Flenner and Grzegorz Szamel. Active matter: quantifying the departure from equilibrium. arXiv preprint arXiv:2004.11925, 2020.

[58] Isabella Petrelli, Leticia F. Cugliandolo, Giuseppe Gonnella, and Antonio Suma. Effective temperatures in inhomogeneous passive and active bidimensional brownian particle systems. arXiv preprint arXiv:2005.02303, 2020.

[59] Roberto Di Leonardo, Luca Angelani, Dario Dell'Arciprete, Giancarlo Ruocco, Valerio Iebba, Serena Schippa, Maria Pia Conte, Francesco Mecarini, Francesco De Angelis, and Enzo 
Di Fabrizio. Bacterial ratchet motors. Proceedings of the National Academy of Sciences, 107(21):9541-9545, 2010.

[60] Andrey Sokolov, Mario M. Apodaca, Bartosz A. Grzybowski, and Igor S. Aranson. Swimming bacteria power microscopic gears. Proceedings of the National Academy of Sciences, 107(3):969 974, 2010.

[61] Héctor Matías López, Jérémie Gachelin, Carine Douarche, Harold Auradou, and Eric Clément. Turning bacteria suspensions into superfluids. Physical Review Letters, 115(2):028301, 2015.

[62] Salima Rafaï, Levan Jibuti, and Philippe Peyla. Effective viscosity of microswimmer suspensions. Physical Review Letters, 104(9):098102, 2010.

[63] Walter F. Paxton, Shakuntala Sundararajan, Thomas E. Mallouk, and Ayusman Sen. Chemical locomotion. Angewandte Chemie International Edition, 45(33):5420-5429, 2006.

[64] Jérémie Palacci, Cécile Cottin-Bizonne, Christophe Ybert, and Lydéric Bocquet. Sedimentation and effective temperature of active colloidal suspensions. Physical Review Letters, 105:088304, Aug 2010.

[65] Jérémie Palacci, Stefano Sacanna, Asher Preska Steinberg, David J. Pine, and Paul M. Chaikin. Living crystals of light-activated colloidal surfers. Science, 339(6122):936-940, 2013.

[66] Isaac Theurkauff, Cécile Cottin-Bizonne, Jérémie Palacci, Christophe Ybert, and Lydric Bocquet. Dynamic clustering in active colloidal suspensions with chemical signaling. Physical Review Letters, 108:268303, Jun 2012.

[67] Sara Dal Cengio, Demian Levis, and Ignacio Pagonabarraga. Linear response theory and greenkubo relations for active matter. Physical Review Letters, 123:238003, Dec 2019.

[68] Nico G. Van Kampen. Langevin-like equation with colored noise. Journal of Statistical Physics, 54(5):1289-1308, Mar 1989.

[69] Hong Qian. Vector field formalism and analysis for a class of thermal ratchets. Physical Review Letters, 81:3063-3066, Oct 1998.

[70] Hong Qian, Min Qian, and Xiang Tang. Thermodynamics of the general diffusion process: Time-reversibility and entropy production. Journal of Statistical Physics, 107(5):1129-1141, Jun 2002.

[71] Jin Wang, Li Xu, and Erkang Wang. Potential landscape and flux framework of nonequilibrium networks: Robustness, dissipation, and coherence of biochemical oscillations. Proceedings of the National Academy of Sciences, 105(34):12271-12276, 2008.

[72] Denis J. Evans and Gary Morriss. Statistical Mechanics of Nonequilibrium Liquids. Cambridge University Press, 2 edition, 2008.

[73] Crispin Gardiner. Stochastic methods. Springer Berlin, 2009.

[74] Julien Tailleur, Sorin Tnase-Nicola, and Jorge Kurchan. Kramers equation and supersymmetry. Journal of Statistical Physics, 122(4):557-595, 2006.

[75] Jorge Kurchan. Six out of equilibrium lectures. arXiv preprint arXiv:0901.1271, 2009.

[76] Matteo Polettini. Of dice and men. subjective priors, gauge invariance, and nonequilibrium thermodynamics. arXiv preprint arXiv:1307.2057, 2013.

[77] Matthias Fuchs and Michael E. Cates. Integration through transients for brownian particles under steady shear. Journal of Physics: Condensed Matter, 17(20):S1681, 2005.

[78] Massimo Falcioni, Stefano Isola, and Angelo Vulpiani. Correlation functions and relaxation properties in chaotic dynamics and statistical mechanics. Physics Letters A, 144(6):341 - 346, 1990.

[79] Leticia F. Cugliandolo, Jorge Kurchan, and Giorgio Parisi. Off equilibrium dynamics and aging in unfrustrated systems. Journal de Physique I, 4(11):1641-1656, 1994.

[80] Hao Ge. Time reversibility and nonequilibrium thermodynamics of second-order stochastic processes. Physical Review E, 89:022127, Feb 2014.

[81] Marco Baiesi, Eliran Boksenbojm, Christian Maes, and Bram Wynants. Nonequilibrium linear response for markov dynamics, ii: Inertial dynamics. Journal of Statistical Physics, 139:492505,2010 . 
[82] Nahuel Freitas, Jean-Charles Delvenne, and Massimiliano Esposito. Stochastic and quantum thermodynamics of driven rlc networks. arXiv preprint arXiv:1906.11233, 2019.

[83] Hong Qian. Mesoscopic nonequilibrium thermodynamics of single macromolecules and dynamic entropy-energy compensation. Physical Review E, 65:016102, Dec 2001.

[84] Udo Seifert. Entropy production along a stochastic trajectory and an integral fluctuation theorem. Physical Review Letters, 95:040602, Jul 2005.

[85] Hao Ge. Extended forms of the second law for general time-dependent stochastic processes. Physical Review E, 80:021137, Aug 2009.

[86] Massimiliano Esposito and Christian Van den Broeck. Three detailed fluctuation theorems. Physical Review Letters, 104:090601, Mar 2010.

[87] Christian Van den Broeck and Massimiliano Esposito. Three faces of the second law. ii. fokkerplanck formulation. Physical Review E, 82:011144, Jul 2010.

[88] Richard E. Spinney and Ian J. Ford. Entropy production in full phase space for continuous stochastic dynamics. Physical Review E, 85:051113, May 2012.

[89] Haidong Feng and Jin Wang. Potential and flux decomposition for dynamical systems and nonequilibrium thermodynamics: Curvature, gauge field, and generalized fluctuation-dissipation theorem. The Journal of Chemical Physics, 135(23):234511, 2011.

[90] Alexandre P. Solon, Michael E. Cates, and Julien Tailleur. Active brownian particles and runand-tumble particles: A comparative study. The European Physical Journal Special Topics, 224(7):1231-1262, 2015.

[91] Peter Hänggi and Peter Jung. Colored noise in dynamical systems. Advances in chemical physics, 89:239-326, 1994.

[92] Claudio Maggi, Umberto Marini Bettolo Marconi, Nicoletta Gnan, and Roberto Di Leonardo. Multidimensional stationary probability distribution for interacting active particles. Scientific reports, 5:10742, 2015.

[93] René Wittmann, Claudio Maggi, Abhinav Sharma, Alberto Scacchi, Joseph M. Brader, and Umberto Marini Bettolo Marconi. Effective equilibrium states in the colored-noise model for active matter i. pairwise forces in the fox and unified colored noise approximations. Journal of Statistical Mechanics: Theory and Experiment, page 113207, 2017.

[94] Ronald F. Fox. Uniform convergence to an effective fokker-planck equation for weakly colored noise. Physical Review A, 34(5):4525, 1986.

[95] Ronald F. Fox. Functional-calculus approach to stochastic differential equations. Physical Review A, 33(1):467, 1986.

[96] Thomas F. Farage, Philip Krinninger, and Joseph M. Brader. Effective interactions in active brownian suspensions. Physical Review E, 91(4):042310, 2015.

[97] René Wittmann and Joseph M. Brader. Active brownian particles at interfaces: An effective equilibrium approach. EPL (Europhysics Letters), 114(6):68004, jun 2016.

[98] Markus Rein and Thomas Speck. Applicability of effective pair potentials for active brownian particles. The European Physical Journal E, 39(9):84, 2016.

[99] Harold Grad. Principles of the Kinetic Theory of Gases, pages 205-294. Springer Berlin Heidelberg, Berlin, Heidelberg, 1958.

[100] Rodrigo Soto. Kinetic Theory and Transport Phenomena. Oxford master series in condensed matter physics. Oxford University Press, 2016. 


\section{Appendix A: Chapman-Enskog method for one dimensional AOUP}

Here we report some details of the Chapman-Enskog procedure for a one dimensional system of AOUP. The starting point is the evolution equation (100) together with the ansatz of Eqs. (102, 103). By integrating (100) over $p$ we obtain a conservation equation for $R$ :

$$
\partial_{t} R=-\partial_{x} \sum_{j} J^{(j)}=-\sum_{j} \epsilon^{j} \partial_{x} \int d p p \phi^{(j)} \equiv \sum_{j} \epsilon^{j} \mathcal{F}^{(j)}
$$

where $J=\sum_{j} J^{(j)}$ is the probability flux.

We now substitute 112 into 100 and we obtain to first order in $\epsilon$ an equation for $\phi^{(1)}$ :

$$
\mathcal{O}(\epsilon): \mathcal{L}\left[\phi^{(1)}\right] \equiv \partial_{p}\left(p \phi^{(1)}+\partial_{p} \phi^{(1)}\right)=p \frac{e^{-p^{2} / 2}}{\sqrt{2 \pi}}\left(\partial_{x} R+U^{\prime} R\right)
$$

In eq. 113 $\phi^{(1)}$ has a gaussian dependence in momenta and we find:

$$
\phi^{(1)}=-p \frac{e^{-p^{2} / 2}}{\sqrt{2 \pi}} \mathcal{D} R
$$

with $\mathcal{D} R \equiv U^{\prime} R+\partial_{x} R$. Note that $\mathcal{L}\left[e^{-p^{2} / 2}\right]=0$ such that the functions $\phi^{(j)}$ are defined up to a constant in $p$ which is uniquely determined by conditions (103). The second order in $\epsilon$ reads:

$$
\mathcal{O}\left(\epsilon^{2}\right): \mathcal{L}\left[\phi^{(2)}\right]=\frac{e^{-p^{2} / 2}}{\sqrt{2 \pi}}\left[\mathcal{F}^{(1)}(R)+\left(p^{2}-1\right) U^{\prime \prime} R\right]+p \partial_{x} \phi^{(1)}-U^{\prime} \partial_{p} \phi^{(1)}(11
$$

From eq. 114 and 115 it follows that $\phi^{(2)}$ is even in p, such that $\mathcal{F}^{(2)}=\int d p p \phi^{(2)}=0$. Using

$$
\mathcal{L}\left[p^{2} \frac{e^{-p^{2} / 2}}{\sqrt{2 \pi}}\right]=-2\left(p^{2}-1\right) \frac{e^{-p^{2} / 2}}{\sqrt{2 \pi}}
$$

we find:

$$
\phi^{(2)}=\alpha(x)\left(p^{2}-1\right) \frac{e^{-p^{2} / 2}}{\sqrt{2 \pi}}
$$

with $\alpha=\frac{1}{2}\left(\partial_{x} \mathcal{D} R+U^{\prime} \mathcal{D} R-U^{\prime \prime} R\right)$.

To third order in $\epsilon$ the evolution equation gives:

$$
\mathcal{O}\left(\epsilon^{3}\right): \mathcal{L}\left[\phi^{(3)}\right]=\frac{\delta \phi^{(1)}}{\delta R} \mathcal{F}^{(1)}(R)+p \partial_{x} \phi^{(2)}-U^{\prime} \partial_{p} \phi^{(2)}-U^{\prime \prime} \partial_{p}\left(p \phi^{(1)}\right)
$$

which, using (114) and (117), together with the identity:

$$
\mathcal{L}\left[p^{3} \frac{e^{-p^{2} / 2}}{\sqrt{2 \pi}}\right]=\frac{e^{-p^{2} / 2}}{\sqrt{2 \pi}} 3 p\left(2-p^{2}\right)
$$

gives:

$$
\phi^{(3)}=p \frac{e^{-p^{2} / 2}}{\sqrt{2 \pi}}\left(\beta(x) p^{2}+\gamma(x)\right)
$$


with $\beta=-\frac{1}{3}\left(\mathcal{D} \alpha-U^{\prime \prime} \mathcal{D} R\right)$ and $\gamma=\mathcal{D} \partial_{x} \mathcal{D} R-\partial_{x} \alpha+U^{\prime} \alpha$. We truncate the hierarchy of equations to $\sim o\left(\epsilon^{3}\right)$ and, going back to the conservative equation (112), we find an expression for the first three components of the flux as a function of $R$ :

$$
\begin{aligned}
J^{(1)} & =-\mathcal{D} R \\
J^{(2)} & =0 \\
J^{(3)} & =\partial_{x} U^{\prime \prime} R
\end{aligned}
$$

We have therefore a closed approximated equation for the evolution of $R$ :

$$
\frac{\partial R}{\partial t}=-\epsilon \partial_{x}\left[-\partial_{x} R-U^{\prime} R+\epsilon^{2} \partial_{x}\left(U^{\prime \prime} R\right)\right]+o\left(\epsilon^{3}\right)
$$

Eq. (124) is readily solved by looking at the zero-flux steady-state with solution:

$$
R(x ; \epsilon)=\exp \left[-U-\epsilon^{2}\left(\frac{1}{2} U^{\prime 2}-U^{\prime \prime}\right)\right]+o\left(\epsilon^{3}\right)
$$

In turn 125 is used to find the explicit expressions of $\phi^{1,2,3}$ which are substituted in (102) to get:

$$
\Psi_{0}(x, p ; \epsilon)=\Psi^{(0)}\left[1+\frac{\epsilon^{2}}{2}\left(1-p^{2}\right) U^{\prime \prime}+\epsilon^{3}\left(\frac{p^{3}}{6} U^{\prime \prime \prime}-\frac{p}{2} U^{\prime \prime \prime}\right)\right]+o\left(\epsilon^{3}\right)
$$

Finally, we use 125 into 126 and interpret the term in square brakets as the linearization of an $\operatorname{exponential~} \exp (x) \sim 1+x$ so that:

$$
\Psi_{0}(x, p ; \epsilon) \sim \exp \left[-\frac{p^{2}}{2}-U-\frac{\epsilon^{2}}{2}\left(p^{2} U^{\prime \prime}+U^{\prime 2}-3 U^{\prime \prime}\right)+\frac{\epsilon^{3}}{2}\left(\frac{p^{3}}{3} U^{\prime \prime \prime}-p U^{\prime \prime \prime}\right)\right]+o\left(\epsilon^{3}\right)(12
$$

